\title{
COMPACTIFICATION AND TREES OF SPHERES COVERS
}

\author{
MATTHIEU ARFEUX
}

\begin{abstract}
The space of dynamically marked rational maps can be identified with a subspace of the space of covers between trees of spheres on which there is a notion of convergence that makes it sequentially compact. In this paper we describe a topology on the quotient of this space under the natural action of its group of isomorphisms. This topology is proved to be consistent with this notion of convergence.
\end{abstract}

\section{INTRODUCTION}

We denote by $\mathbb{S}:=\mathbb{P}^{1}(\mathbb{C})$ the Riemann sphere. For $d \geq 1$, we denote by Rat $_{d}$ the space of rational maps $f: \mathbb{S} \rightarrow \mathbb{S}$ of degree $d \geq 1$ endowed with the topology of uniform convergence. In particular, Aut $(\mathbb{S}):=$ Rat $_{1}$ is the group of Moebius transformations and acts on Rat $_{d}$ by conjugacy:

$$
\operatorname{Aut}(\mathbb{S}) \times \operatorname{Rat}_{d} \ni(\phi, f) \mapsto \phi \circ f \circ \phi^{-1} \in \operatorname{Rat}_{d} .
$$

We are interested in the quotient rat $_{d}$ of Rat $d$ by this action. The space rat is not compact for any $d$.

In this paper we provide a compactification of a related space: the moduli space rat $_{\mathbf{F}}$ of rational maps marked by a portrait $\mathbf{F}$ (i.e., the space of rational maps with some prescribed restriction on a finite set; see Definition 2.1). A marked rational map is a triple $(f, y, z)$ where $f \in \operatorname{Rat}_{d}$ and $y: Y \rightarrow \mathbb{S}$ and $z: Z \rightarrow \mathbb{S}$ are injections of finite sets $Y$ and $Z$ with at least three elements (often called marked spheres). This triple satisfies in particular

$$
y(Y)=f^{-1}(z(Z)) .
$$

The maps $y$ and $z$ are representatives of classes in $\operatorname{Mod}_{Y}$ and $\operatorname{Mod}_{Z}$, the respective moduli spaces of spheres marked by $Y$ and $Z$.

We will remark that the projection of $\operatorname{rat}_{\mathbf{F}}$ to $\operatorname{Mod}_{Y}$ is injective, and we use the Deligne-Mumford compactification $\overline{\operatorname{Mod}}_{Y}$ of $\operatorname{Mod}_{Y}$ (cf. [DeM]) to provide a compactification of $\operatorname{rat}_{\mathbf{F}}$. Following $\left[\mathrm{FT}\right.$, we identify $\overline{\operatorname{Mod}}_{Y}$ to the space $\widehat{\operatorname{Mod}}_{Y}$ of isomorphism classes of trees of spheres (cf. also [B]).

The aim of this paper is to remark that we can identify the elements of this compactification to isomorphism classes of covers between trees of spheres of portrait $\mathbf{F}$ (see the definition in Section 4.1). More precisely, we define $\widehat{\operatorname{rat}}_{\mathbf{F}}$ to be the set of such classes of covers $\mathcal{F}: \mathcal{T}^{Y} \rightarrow \mathcal{T}^{Z}$ where $\left[\mathcal{T}^{Y}\right] \in \widehat{\operatorname{Mod}}_{Y}$ and $\left[\mathcal{T}^{Z}\right] \in \widehat{\operatorname{Mod}}_{Z}$.

Received by the editors October 14, 2016, and, in revised form, February 10, 2017.

2010 Mathematics Subject Classification. Primary 37F20.

Key words and phrases. Limits of dynamical systems, compactification, rescaling limits, Deligne-Mumford compactification, algebraic geometry, trees of spheres, noded spheres. 
We endow it with a topology via the map

$$
\text { I : } \widehat{\operatorname{rat}}_{\mathbf{F}} \rightarrow \widehat{\operatorname{Mod}}_{Y}
$$

that maps $[\mathcal{F}]$ to $\left[\mathcal{T}^{Y}\right]$. We identify $\operatorname{rat}_{\mathbf{F}}$ as a subspace of $\widehat{\operatorname{rat}}_{\mathbf{F}}$ and prove:

Theorem 1. The map I is injective and $\widehat{\mathbf{r a t}}_{\mathbf{F}}$ is the compact closure of $\operatorname{rat}_{\mathbf{F}}$.

As a byproduct, when the portrait allows dynamics, i.e., when there exists a nontrivial set $X \subset Y \cap Z$, the compactification allows dynamics on trees of spheres. We define the notion of trees of spheres dynamical systems (see the definition in Section 5.1) together with a natural, but more restrictive, notion of isomorphism. We explain how to deduce the compactness of the space of isomorphism classes of trees of spheres dynamical systems from the one of $\widehat{\mathbf{r a t}}_{\mathbf{F}}$.

This paper will set up the topological ground for the study of the degeneracy of dynamical systems on the Riemann sphere. The works [A1 and A2 begin this study and present the motivations for introducing trees of spheres dynamical systems in this context.

Outline. In Section 2, we make clear what we mean by portrait, rational map marked or dynamically marked by a portrait, and what are the natural isomorphism classes associated to these maps.

Section 3 is devoted to a deepening of the work of R. Funahashi and M. Taniguchi in [FT]. For any finite set $E$ with at least three elements, we identify $\overline{\operatorname{Mod}}_{E}$ to the moduli space $\widehat{\operatorname{Mod}}_{E}$ endowed with a topology that also makes it compact. In addition, we define the notion of convergence of a sequence of marked spheres to a tree of spheres which is compatible with this topology.

In Section 4, we identify $\operatorname{rat}_{\mathbf{F}}$ as a subspace of $\widehat{\mathbf{r a t}}_{\mathbf{F}}$ and prove Theorem 1] We also define the notion of convergence of a sequence of marked spheres covers to a cover between trees of spheres which is compatible with the ambient topology.

In Section 5, we identify $\operatorname{rat}_{\mathbf{F}, X}$ with a subspace of $\operatorname{rat}_{\mathbf{F}}$. We introduce $\mathbf{d y n}_{\mathbf{F}, X}$ as the set of dynamical systems between trees of spheres modulo a certain notion of isomorphism and prove that it can also be identified with a subspace of $\widehat{\mathbf{r a t}}_{\mathbf{F}}$. We deduce that $\mathbf{d y} \mathbf{n}_{\mathbf{F}, X}$ is compact and prove that its topology is compatible with a natural notion of dynamical convergence. We show that the discussions in A2 prove that for some $\mathbf{F}$ we can have:

$$
\overline{\operatorname{rat}}_{\mathbf{F}, X} \subsetneq \mathbf{d y n} \mathbf{F , X} \subsetneq \widehat{\operatorname{rat}}_{\mathbf{F}} .
$$

\section{MARKING RATIONAL MAPS}

In this section, $X, Y$ and $Z$ denote finite sets with at least three elements.

2.1. Spaces. For any finite set $E$, a sphere marked by $E$ is an injection $E \rightarrow \mathbb{S}$. A portrait $\mathbf{F}$ of degree $d \geq 2$ is a pair $(F, \operatorname{deg})$ where

- $F: Y \rightarrow Z$ is a map between $Y$ and $Z$, and

- $\operatorname{deg}: Y \rightarrow\{1,2, \ldots, d\}$ is a function that satisfies

$$
\sum_{a \in Y}(\operatorname{deg}(a)-1)=2 d-2 \quad \text { and } \quad \sum_{a \in F^{-1}(b)} \operatorname{deg}(a)=d \quad \text { for all } b \in Z \text {. }
$$


Typically, $Z \subset \mathbb{S}$ is a finite set, $F: Y \rightarrow Z$ is the restriction of a rational map $f: \mathbb{S} \rightarrow \mathbb{S}$ to $Y:=f^{-1}(Z)$ of degree $d$ and $\operatorname{deg}(a)$ is the local degree of $f$ at $a$. In this case, the Riemann-Hurwitz formula and the conditions on the function deg implies that $Z$ contains the set of critical values of $f$ so that $f: \mathbb{S}-Y \rightarrow \mathbb{S}-Z$ is an unbranched cover.

Definition 2.1. A rational map marked by a degree $d$ portrait $\mathbf{F}$ is a triple $(f, y, z)$ where

- $f \in \operatorname{Rat}_{d}$,

- $y: Y \rightarrow \mathbb{S}$ and $z: Z \rightarrow \mathbb{S}$ are marked spheres,

- $f \circ y=z \circ F$ on $Y$ and

- $\operatorname{deg}_{y(a)} f=\operatorname{deg}(a)$ for $a \in Y$.

Note that in particular, from the definition of $\mathbf{F}$ it follows that $y(Y)=f^{-1}(z(Z))$.

Definition 2.2. A rational map dynamically marked by $(\mathbf{F}, X)$ is a triple $(f, y, z)$ which is a rational map marked by $\mathbf{F}$ such that $X \subseteq Y \cap Z$ and $\left.y\right|_{X}=\left.z\right|_{X}$.

We denote by Rat $\mathbf{F}$ the set of rational maps marked by $\mathbf{F}$ and $\operatorname{Rat}_{\mathbf{F}, X}$ the set of rational maps dynamically marked by $(\mathbf{F}, X)$.

2.2. Quotient spaces. The group $\operatorname{Aut}(\mathbb{S})$ acts on Rat Fy pre-composition and post-composition: a pair of Moebius transformations $(\phi, \psi) \in \operatorname{Aut}(\mathbb{S}) \times \operatorname{Aut}(\mathbb{S})$ maps the marked rational map $(f, y, z) \in \operatorname{Rat}_{\mathbf{F}}$ to

$$
\left(\phi \circ f \circ \psi^{-1}, \psi \circ y, \phi \circ z\right) \in \operatorname{Rat}_{\mathbf{F}}
$$

as in the following diagram:

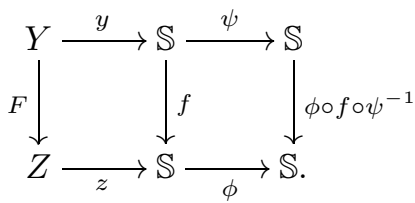

We denote by $\operatorname{rat}_{\mathbf{F}}$ the quotient of $\operatorname{Rat}_{\mathbf{F}}$ by the action of $\operatorname{Aut}(\mathbb{S}) \times \operatorname{Aut}(\mathbb{S})$.

Likewise, the group Aut(S) acts on $\operatorname{Rat}_{F, X}$ by conjugacy: a Moebius transformation $\phi \in \operatorname{Aut}(\mathbb{S})$ maps the dynamically marked rational map $(f, y, z) \in \operatorname{Rat}_{\mathbf{F}, X}$ to

$$
\left(\phi \circ f \circ \phi^{-1}, \phi \circ y, \phi \circ z\right) \in \operatorname{Rat}_{\mathbf{F}, X} .
$$

We denote by $\operatorname{rat}_{\mathbf{F}, X}$ the quotient of $\operatorname{Rat}_{\mathbf{F}, X}$ by the action of $\operatorname{Aut}(\mathbb{S})$.

For any finite set $E$ with at least three elements, we define the moduli space $\operatorname{Mod}_{E}$ to be the space of spheres marked by $E$ modulo post-composition by Moebius transformations.

As $\operatorname{card} X \geq 3$ and if $(f, y, z) \in \operatorname{Rat}_{\mathbf{F}}$, then $f$ is determined by the pair $(y, z)$. Indeed, a rational map is totally determined if we know the preimages, with multiplicities, of any triple of points. Thus $\operatorname{rat}_{\mathbf{F}, X}$ naturally injects into the product of the moduli space of spheres marked by $Y$ and by $Z$,

$$
\operatorname{rat}_{\mathbf{F}, X} \rightarrow \operatorname{Mod}_{Y} \times \operatorname{Mod}_{Z}
$$

In fact we will prove that the natural projection

$$
\operatorname{rat}_{\mathbf{F}} \rightarrow \operatorname{Mod}_{Y}
$$

is also injective, i.e., $[y] \in \operatorname{Mod}_{Y}$ characterizes $[(f, y, z)] \in \operatorname{rat}_{\mathbf{F}}$. 

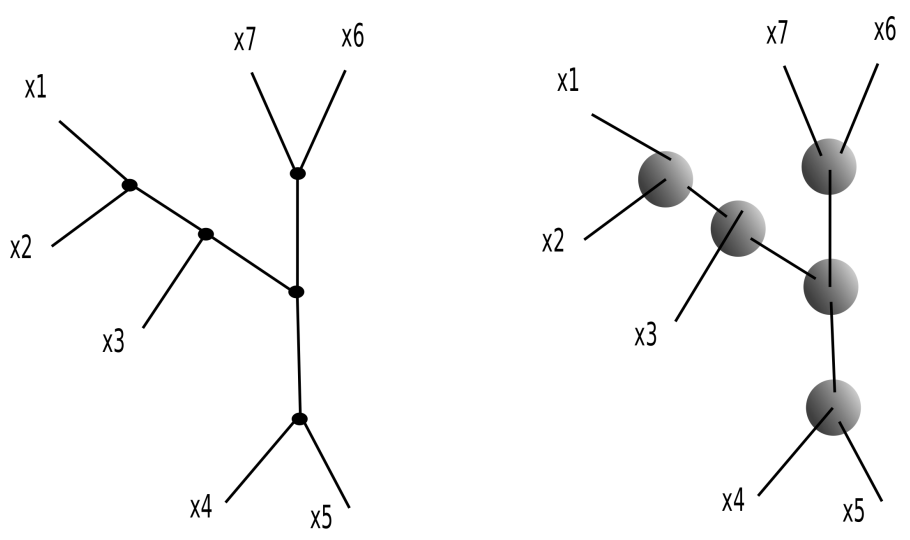

Figure 1. On the left, a combinatorial tree $T^{X}$ for $X=\{x 1 \ldots x 7\}$. On the right, the representation of a tree of spheres whose combinatorial tree is $T^{X}$.

\section{ISOMORPHISM CLASSES OF TREES OF SPHERES}

In this section, $X$ denotes a finite set with at least three elements.

3.1. Trees of spheres. In this subsection we recall notions and notation introduced in [A1].

A (projective) tree of spheres $\mathcal{T}$ marked by $X$ (or $\mathcal{T}^{X}$, cf. example on Figure 1) is the following data:

- a combinatorial tree $T$ ( or $T^{X}$ ) marked by $X$, disjoint union of its set of vertices $V$ and its set of edges $E$, whose set of leaves (external vertices) is $X$ and such that every internal vertex has at least valence 3 (stability), and

- for each internal vertex $v$ of $T$, an injection $i_{v}: E_{v} \rightarrow \mathbb{S}_{v}$ of the set $E_{v}$ of edges adjacent to $v$ into a projective sphere $\mathbb{S}_{v}$.

We denote by $\left\{v, v^{\prime}\right\}$ the edge between two vertices $v$ and $v^{\prime}$ if it exists. We use the notation $X_{v}:=i_{v}\left(E_{v}\right)$ and define the map $a_{v}: X \rightarrow \mathbb{S}_{v}$ such that $a_{v}(x):=i_{v}(e)$ if $x$ and $e$ lie in the same connected component of $T-\{v\}$. We say that $i_{v}(e)$ is the attaching point of the edge $e$ on $v$ or $\mathbb{S}_{v}$.

For $\star \in T \backslash\{v\}$ we denote by $B_{v}(\star)$ the connected component of $T \backslash\{v\}$ containing $\star$. It is called the branch at $v$ containing $\star$. For any vertex $v^{\prime}$ in a branch $B_{v}(e)$ where $e$ is an edge adjacent to $v$, we will also make use of the notation $i_{v}\left(v^{\prime}\right)$ for the attaching point $i_{v}(e)$.

We denote by $\left[v, v^{\prime}\right]$ the arc (i.e., injective path) between $v$ and $v^{\prime}$. We will also denote it by $\left[v_{1}, v_{2}, \ldots, v_{k}\right]$ if $v=v_{1}, v^{\prime}=v_{k}$ and the vertices $v_{i}$ are adjacent to the $v_{i+1}$.

\subsection{Isomorphism of combinatorial trees and partitions.}

Definition 3.1 (Tree maps). A (combinatorial) tree map is a map between two combinatorial trees that maps internal vertices to internal vertices, external ones to external ones, edges to edges and two adjacent vertices to adjacent ones. 
Definition 3.2 (Isomorphism of marked trees). An isomorphism between two combinatorial trees marked by $X$ is a tree map which is bijective and restricts to the identity on $X$.

Define $P_{v}:=\left\{B_{v}(e) \cap X \mid e \in E_{v}\right\}$. By convention, a partition does not contain the element $\emptyset$. For all $v$ internal vertex, $P_{v}$ is a partition of $X$.

Let $\psi$ be the map between the set of trees marked by $X$ and the set of collections of partitions of $X$ that maps $T$ to

$$
\psi(T)=\left\{P_{v} \subset X \mid v \text { internal vertex of } T^{X}\right\} .
$$

The goal of this section is to give a characterization of the image of this map and of the isomorphism classes of combinatorial trees marked by $X$.

Definition 3.3 (Admissible collection of partitions). A collection $\mathcal{P}$ of partitions is admissible if it satisfies the following properties:

(1) every partition $P \in \mathcal{P}$ contains at least three distinct elements,

(2) for all partition $P \in \mathcal{P}$ and all subset $B \in P$, either there exists a partition $P^{\prime} \in \mathcal{P}$ containing $X-B$, or $B=\{x\}$ with $x \in X$,

(3) if $P_{1} \in \mathcal{P}$ and $P_{2} \in \mathcal{P}$ are two distinct partitions, then $P_{1} \cap P_{2}=\emptyset$.

Remark 3.4. If $T$ is a combinatorial tree, then $\psi(T)$ is clearly a collection of admissible partitions (a detailed proof can be found in $[\mathrm{A}]$ ).

We prove the following theorem:

Theorem 3.5. Two combinatorial trees $T$ and $T^{\prime}$ are isomorphic if and only if $\psi(T)=\psi\left(T^{\prime}\right)$. If $T$ is a combinatorial tree, then $\psi(T)$ is a collection of admissible partitions, and every admissible collection of partition is the image of a (stable) tree.

Corollary 3.6. The map $\psi$ induces a bijection between the set of isomorphism classes of trees and the set of admissible collections of partitions.

We finish this section by the proof of Theorem 3.5 .

Take any admissible collection of partitions $\mathcal{P}$. Define a set of vertices $V_{T}=\mathcal{P} \cup X$. Define a set of edges $E_{T}$ as the set of $\left\{P_{1}, P_{2}\right\}$ for all $P_{1} \in \mathcal{P}$ and $P_{2} \in \mathcal{P}$ such that we have $B_{i} \in P_{1}$ and $B_{2} \in P_{2}$ satisfying $B_{1} \cup B_{2}=X$ with $B_{1} \cap B_{2}=\emptyset$ and the $\left\{P_{0}, x\right\}$ satisfying $P_{0} \in \mathcal{P}$ and $\{x\} \in P_{0}$. We define the graph $T_{\mathcal{P}}$ to be $V_{T} \sqcup E_{T}$.

Lemma 3.7. Let $x \in X$. Every vertex $v \in V_{T} \backslash\{x\}$ can be connected to the vertex $x$ by a unique arc. Moreover, if the first edge of this arc is $\{v, P\}$, then $x \in P$.

Proof. Define $v_{1}:=v$. We are looking for an $\operatorname{arc}\left[v_{1}, v_{2}, v_{3}, \ldots, v_{k}, x\right]$.

If $v_{1} \in X$, then the second property assures the existence of a vertex $v_{2}$ such that $\left\{v_{1}\right\}$ lies in the partition. Then we are in the case $v_{1} \notin X$. We find the $v_{i}$ recursively.

Recurrence hypothesis: there exist $v_{2}, \ldots, v_{i}$ such that $\left[v_{1}, v_{2}, \ldots, v_{i}\right]$ is an arc and the element $B_{i}$ of $v_{i}$ containing $x$ is included in the one of $v_{i-1}$ containing $x$.

Suppose that it is true for some $i \in \mathbb{N}$. Let $B_{i}$ be this element. If $B_{i}=\{x\}$, then by construction $\left\{v_{i},\{x\}\right\} \in E_{T}$ and $\left[v_{1}, v_{2}, \ldots, v_{i},\{x\}\right]$ is the desired arc. If not, we find $v_{i+1}$ containing $X \backslash B_{i} \in V_{T}$. Thus $\left\{v_{i}, v_{i+1}\right\} \in E_{T}$. If $B_{i+1}$ is the subset of $v_{i+1}$ containing $x$, then $B_{i+1}$ and $X \backslash B_{i}$ are two elements of the partition $v_{i+1}$ so we have $B_{i+1} \subset B_{i}$ as desired. The property is true for $i+1$. 
This construction stops because the inclusions of $B_{i}$ are strict. In addition we always have $x \in B_{i}$. It follows that if $v_{k}$ is the last vertex of the constructed arc, then $v_{k}=x$.

This arc is unique because the hypothesis $B_{i+1} \subset B_{i}$ is necessary and induces the unicity for the choice of the vertices choices at every step. The end of the lemma follows from the construction.

Corollary 3.8. The graph $T_{\mathcal{P}}$ is a combinatorial tree marked by $X$ and $\psi\left(T_{\mathcal{P}}\right)=\mathcal{P}$.

Proof. For $T_{\mathcal{P}}$, the connectivity and the non-existence of cycle is clear from Lemma 3.7. The stability is direct from the first property of Definition 3.3. and by construction the leaves of our tree are the elements of $X$. Hence $T_{\mathcal{P}}$ is a combinatorial tree marked by $X$.

Now we prove that $\psi\left(T_{\mathcal{P}}\right)=\mathcal{P}$. Let $v_{1} \in V_{T}$. Denote by $P=\left\{p_{1}, \ldots, p_{k}\right\}$ the associated partition at the edges $\left\{p_{i}, \star\right\}$ of $v_{1}$. From Lemma 3.7 deduce that $B_{v_{1}}\left(\left\{p_{i}, \star\right\}\right) \subseteq p_{1}$. But $P$ is a partition so it is an equality.

Thus we proved the following:

Corollary 3.9. The map $\psi$ is surjective onto the set of admissible collections of partitions.

Proof. (Theorem 3.5) Remark 3.4 and Corollary 3.9 prove the two first statements.

As an isomorphism between combinatorial trees is a tree map, we deduce that it maps an arc to an arc, so a branch to a branch, and it follows that two isomorphic combinatorial trees have the same image by $\phi$.

Suppose that two combinatorial trees $T$ and $T^{\prime}$ have the same image by $\phi$. Let us prove that $T$ and $T^{\prime}$ are isomorphic. Any internal vertex $v \in T$ has at least three branches and every branch contains at least one element of $X$. Take three elements of $X$ lying in three different branches of $v$. Then there exists a unique internal vertex $v^{\prime} \in T^{\prime}$ that has these three elements in different branches. Let us define $M(v):=v^{\prime}$. As $\phi(T)=\phi\left(T^{\prime}\right)$, this does not depend on the choice of the elements in $X$. For $x \in X$ we define $M(x):=x$.

Let us prove that $M$ is a tree map. For simplicity we are going to suppose that $T^{\prime}=T_{\phi(T)}$. Take that $v$ and $w$ are two adjacent vertices of $T$. Suppose that neither $v$ nor $w$ are leaves. Then it is clear that $B_{v}(w)=X \backslash B_{w}(v)$. Because $M(v)$ and $M(w)$ give the same partitions of $X$ as $v$ and $w$, the construction of $T_{\phi(T)}$ ensures that $M(v)$ and $M\left(v^{\prime}\right)$ are adjacent. The case when $v$ or $w$ is a leaf is similar.

\subsection{Isomorphism of trees of spheres and topology.}

Definition 3.10 (Isomorphism of trees of spheres). An isomorphism $\mathcal{M}: \mathcal{T}_{1} \rightarrow \mathcal{T}_{2}$ between two trees of spheres marked by $X$ is an isomorphism $M: T_{1} \rightarrow T_{2}$ between the corresponding combinatorial trees and for every internal vertex $v \in T_{1}$, a (projective) isomorphism $m_{v}: \mathbb{S}_{v} \rightarrow \mathbb{S}_{M(v)}$ that maps the attaching point of an adjacent edge $e$ to the attaching point of $M(e)$.

Let $\mathfrak{T}_{X}$ be the set of trees of spheres marked by $X$. We define on $\mathfrak{T}_{X}$ an equivalence relation given by: $\mathcal{T} \sim \mathcal{T}^{\prime}$ if and only if there exists an isomorphism $\mathcal{M}: \mathcal{T} \rightarrow \mathcal{T}^{\prime}$ of trees of spheres marked by $X$. Note that it follows that for all internal vertex $v$ of $T, m_{v}: \mathbb{S}_{v} \rightarrow \mathbb{S}_{M(v)}$ is an isomorphism and $a_{M(v)}=m_{v} \circ a_{v}$. We will sometimes use the notation $T \sim_{\mathcal{M}} T^{\prime}$. 
We let moduli space of trees of spheres be marked by $X$ and denote by $\widehat{\operatorname{Mod}}_{X}$ the quotient of the set $\mathfrak{T}_{X}$ by this equivalence relation. We denote by $\operatorname{Mod}_{X}$ the set of isomorphism classes of trees of spheres with a unique internal vertex marked by $X$.

If $v$ is the unique vertex of $\mathcal{T} \in \operatorname{Mod}_{X}$, then $\mathcal{T}$ is determined by the element $\left[a_{v}\right] \in \operatorname{Mod}_{X}$. Reversely, given an element $[i: X \rightarrow \mathbb{S}] \in \operatorname{Mod}_{X}$, we can define a tree $\mathcal{T}$ marked by $X$ that has a unique internal vertex $v$ with $\mathbb{S}_{v}=\mathbb{S}$ and $a_{v}=i$. We will freely consider these elements in $\operatorname{Mod}_{X}$ or $\operatorname{Mod}_{X}$ according to convenience.

Let Quad $_{X}$ denote the set of quadruples of distinct elements of $X$. As in [FT], we consider the embedding:

$$
\mathfrak{B}_{X}: \operatorname{Mod}_{X} \rightarrow \mathbb{S}^{\text {Quadx }}
$$

that associates to $[i] \in \operatorname{Mod}_{X}$ the collection of the cross ratios

$$
\left[i\left(x_{1}\right), i\left(x_{2}\right), i\left(x_{3}\right), i\left(x_{4}\right)\right]_{\left(x_{1}, x_{2}, x_{3}, x_{4}\right) \in \operatorname{Quad}_{\mathrm{X}}} \cdot
$$

Denote by Tripx the set of triples of distinct elements of $X$. Consider a combinatorial tree $T$ marked by $X$. Take $t:=\left(x_{0}, x_{1}, x_{\infty}\right) \in$ Tripx. The vertices $x_{0}, x_{1}$ and $x_{\infty}$ are separated by a unique vertex $v_{t}$. We say that this vertex separates the triple $t$.

If this $T$ is the combinatorial tree of a tree of spheres $\mathcal{T}$, then the map $a_{v_{t}}$ maps the three elements of $t$ to distinct images. So there exists a unique projective chart $\sigma_{t}: \mathbb{S}_{v_{t}} \rightarrow \mathbb{S}$ satisfying $\sigma_{t} \circ a_{v_{t}}\left(x_{0}\right)=0, \sigma_{t} \circ a_{v_{t}}\left(x_{1}\right)=1$ and $\sigma_{t} \circ a_{v_{t}}\left(x_{\infty}\right)=\infty$. The map $\sigma_{t}$ is called the $t$-chart of $\mathcal{T}$.

Define

$$
\alpha_{t}:=\sigma_{t} \circ a_{v_{t}}: X \rightarrow \mathbb{S} .
$$

One can easily check that the composition $\sigma_{t} \circ a_{v_{t}}: X \rightarrow \mathbb{S}$ does not depend on the choice of a representative in the class of $\mathcal{T}$.

Recall that Quadx is the set of quadruples of distinct elements of $X$.

Definition 3.11. We define the following map:

$$
\mathfrak{B}_{X}: \widehat{\operatorname{Mod}}_{X} \rightarrow \mathbb{S}^{\text {Quadx }}
$$

that maps every $[T] \in \widehat{\operatorname{Mod}}_{X}$ to the collection of the $\left(\alpha_{t}(x)\right)_{(t, x) \in \operatorname{Quad}_{\mathbf{X}}}$.

The map $\mathfrak{B}_{X}$ defines a topology on $\widehat{\operatorname{Mod}}_{X}$. We will sometimes simply write $\mathfrak{B}$ when there is no possible confusion. The following lemma implies that this topology is Haussdorff.

Lemma 3.12. The map $\mathfrak{B}$ is injective.

Proof. Let $T$ be a tree of spheres marked by $X$. For a fixed $t \in$ Tripx, the data of $\alpha_{t}(x)$ is sufficient to build the map $a_{v}$ when $t$ is separated by the vertex $v$ of $T$. Every internal vertex $v$ is adjacent to at least three edges, so we can always find an element of Tripx separated by $v$. Thus, Theorem 3.5 assures that the class of $T$ is uniquely determined.

Remark 3.13. It follows that the map $\mathfrak{B}$ is a homeomorphism onto its image that equips $\operatorname{Mod}_{X}$ with a quasi-projective variety structure which is the same as the one of $\operatorname{Mod}_{X}$ (via the identification). 
3.4. Convergence notion. We define as in A1 the notion of convergence of a sequence of marked spheres to a marked tree of spheres as follows.

Definition 3.14 (Convergence of marked spheres). A sequence of marked spheres $x_{n}: X \rightarrow \mathbb{S}_{n}$ converges to a tree of spheres $\mathcal{T}^{X}$ if for all internal vertex $v$ of $\mathcal{T}^{X}$, there exists a (projective) isomorphism $\phi_{n, v}: \mathbb{S}_{n} \rightarrow \mathbb{S}_{v}$ such that $\phi_{n, v} \circ x_{n}$ converges to $a_{v}$.

We will use the notation $x_{n} \underset{\phi_{n}}{\longrightarrow} \mathcal{T}^{X}$ or simply $x_{n} \rightarrow \mathcal{T}^{X}$. This convergence notion is compatible with the topology.

Lemma 3.15. Let $\left(\mathcal{T}_{n}\right)_{n}$ and $\left(\mathcal{T}_{n}^{\prime}\right)_{n}$ be two sequences of spheres marked by $X$ and let $\mathcal{T}$ and $\mathcal{T}^{\prime}$ be two trees of spheres marked by $X$.

(1) (quotient)

- if $\mathcal{T} \sim \mathcal{T}^{\prime}$, then $\mathcal{T}_{n} \rightarrow \mathcal{T} \Longleftrightarrow \mathcal{T}_{n} \rightarrow \mathcal{T}^{\prime}$,

- if $\mathcal{T}_{n} \sim \mathcal{T}_{n}^{\prime \prime}$, then $\mathcal{T}_{n} \rightarrow \mathcal{T} \Longleftrightarrow \mathcal{T}_{n}^{\prime} \rightarrow \mathcal{T}$.

(2) (unicity of the limit) if $\mathcal{T}_{n} \rightarrow \mathcal{T}$ and $\mathcal{T}_{n} \rightarrow \mathcal{T}^{\prime}$, then $\mathcal{T} \sim \mathcal{T}^{\prime}$.

Proof. If $\mathcal{T}_{n} \rightarrow_{\phi_{n}} \mathcal{T}$ and $\mathcal{T}^{\prime} \sim_{\mathcal{M}} \mathcal{T}$, then $\mathcal{T}_{n} \rightarrow_{\phi_{n}^{\prime}} \mathcal{T}^{\prime}$ with $\phi_{n, v}=m_{v} \circ \phi_{n, v}^{\prime}$. In addition, if $\mathcal{T}_{n} \sim \mathcal{M} \mathcal{T}_{n}^{\prime} \rightarrow_{\phi_{n}^{\prime}} \mathcal{T}$, then $\mathcal{T}_{n} \rightarrow_{\phi_{n}^{\prime} \circ \mathcal{M}} \mathcal{T}$ which concludes the proof of point 1

For point 2, suppose that $\mathcal{T}_{n} \rightarrow_{\phi_{n}} \mathcal{T}$ and $\mathcal{T}_{n} \rightarrow_{\phi_{n}^{\prime}} \mathcal{T}^{\prime}$. For every internal vertex $v$ of $\mathcal{T}$, chose a triple $t \in \operatorname{Trip}_{\mathrm{X}}$ such that $v$ separates $t$. Denote by $v^{\prime}$ the vertex of $\mathcal{T}^{\prime}$ separated by the same $t$. The map $\sigma_{t}^{\prime} \circ \phi_{n, v^{\prime}}^{\prime-1} \circ \phi_{n, v} \circ \sigma_{t}^{-1}$ is a Moebius transformation that fixes 0,1 and $\infty$, so it is the identity. Thus $\phi_{n, v^{\prime}}^{\prime-1} \circ \phi_{n, v}$ converges to an isomorphism $m_{v}$.

Proposition 3.16. The map $\mathfrak{B}$ defines the same convergence notion as the one on trees of spheres on $\operatorname{Mod}_{X}$, i.e.:

$$
\mathcal{T}_{n} \rightarrow \mathcal{T} \text { if and only if } \mathfrak{B}\left(\left[\mathcal{T}_{n}\right]\right) \rightarrow \mathfrak{B}([\mathcal{T}]) .
$$

Proof. Lemma 3.15 assures that these two formulations are equivalent. Suppose that $\mathcal{T}_{n} \underset{\phi_{n}}{\longrightarrow} \mathcal{T}$. Let $t \in$ Tripx. Let $x \in X$ which does not lie in $t$. Let $\sigma_{n, t}$ be the $t$-chart of $\mathcal{T}_{n}$. Let $\sigma_{t}$ be the $t$-chart of $\mathcal{T}$. Let $v$ be the vertex of $\mathcal{T}$ defined by $t$. Then $m_{n}:=\sigma_{t} \circ \phi_{n, v}^{-1} \circ \sigma_{n, t}$ (cf. the following diagram) is a Moebius transformation that fixes 0,1 and $\infty$ so $m_{n}$ is the identity

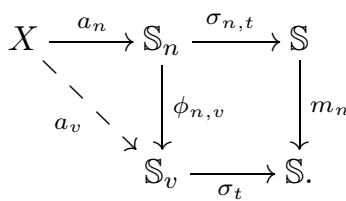

Then we have

$$
\sigma_{n, t} \circ a_{n}(x)=m_{n} \circ \sigma_{n, t} \circ a_{n}(x)=\sigma_{t} \circ \phi_{n, v} \circ a_{n}(x) \rightarrow \sigma_{t} \circ a_{v}(x) .
$$

Thus $\alpha_{n, t} \rightarrow \alpha_{t}$ so $\mathfrak{B}\left(\left[\mathcal{T}_{n}\right]\right) \rightarrow \mathfrak{B}([\mathcal{T}])$.

If in addition $\mathfrak{B}\left(\left[\mathcal{T}_{n}\right]\right) \rightarrow \mathfrak{B}([\mathcal{T}])$, for all internal vertex $v$ of $\mathcal{T}$ denote by $t_{v}$ a triple that defines $v$ and $\sigma_{n, t_{v}}$ the $t_{v}$-chart of $\mathcal{T}_{n}$. Define $\phi_{n, v}:=\sigma_{n, t_{v}}^{-1} \circ \sigma_{t_{v}}$. Then we have $\phi_{n, v} \circ a_{n} \rightarrow a_{v}$. 
The notion of convergence of a sequence of trees of spheres is not used in this paper. Let us however make it explicit in the remark below.

Remark 3.17 (Convergence of trees). Let $\left(\mathcal{T}_{n}\right)_{n}$ be a sequence of trees marked by $X$ and $\mathcal{T} \in \widehat{\operatorname{Mod}}_{X}$. For all $t \in \operatorname{Trip}_{\mathrm{X}}$ we denote by $v_{n, t}$ the vertex of $\mathcal{T}_{n}^{X}$ separating t. By the definition of $\mathfrak{B}$ we know that $\left(\mathcal{T}_{n}\right)_{n}$ converges to $\mathcal{T}$ if

$$
\forall t \in \operatorname{TripX}, \exists \phi_{\mathrm{n}, \mathrm{v}_{\mathrm{n}, \mathrm{t}}} \in \operatorname{Aut}\left(\mathbb{S}_{\mathrm{v}_{\mathrm{n}, \mathrm{t}}}, \mathbb{S}_{\mathrm{v}_{\mathrm{t}}}\right), \quad \phi_{\mathrm{n}, \mathrm{v}_{\mathrm{n}, \mathrm{t}}} \circ \mathrm{a}_{\mathrm{n}, \mathrm{v}_{\mathrm{n}, \mathrm{t}}} \rightarrow \mathrm{a}_{\mathrm{v}} .
$$

To conclude this section, we state the following useful property for which we recall a proof from [A1] to make clear the independence with this latter paper.

Lemma 3.18. Let $v$ and $v^{\prime}$ be two distinct internal vertices of $\mathcal{T}^{X}$, and consider a sequence of marked spheres $\left(x_{n}\right)_{n}$ such that $x_{n} \underset{\phi_{n}}{\longrightarrow} \mathcal{T}^{X}$. Then the sequence of isomorphisms $\left(\phi_{n, v^{\prime}} \circ \phi_{n, v}^{-1}\right)_{n}$ converges locally uniformly outside $i_{v}\left(v^{\prime}\right)$ to the constant $i_{v^{\prime}}(v)$.

Proof. Each vertex $v$ and $v^{\prime}$ has three edges and every branch has at least a leaf, so there exist four marked points $\chi_{1}, \chi_{2}, \chi_{3}, \chi_{4} \in X$ such that $v$ separates $\chi_{1}, \chi_{2}$ and $v^{\prime}$, and the vertex $v^{\prime}$ separates $\chi_{3}, \chi_{4}$ and $v$.

We define for $j \in\{1,2,3,4\}$,

$\xi_{j}:=a_{v}\left(\chi_{j}\right), \quad \xi_{j}^{\prime}:=a_{v^{\prime}}\left(\chi_{j}\right), \quad \xi_{j, n}:=\phi_{n, v} \circ x_{n}\left(\chi_{j}\right) \quad$ and $\quad \xi_{j, n}^{\prime}:=\phi_{n, v^{\prime}} \circ x_{n}\left(\chi_{j}\right)$.

From the hypothesis, $\xi_{j, n} \rightarrow \xi_{j}$ and $\xi_{j, n}^{\prime} \rightarrow \xi_{j}^{\prime}$ when $n \rightarrow \infty$. Moreover, $\xi_{3}=\xi_{4}=$ $i_{v}\left(v^{\prime}\right)$ and $\xi_{1}^{\prime}=\xi_{2}^{\prime}=i_{v^{\prime}}(v)$. After maybe post-composing $\phi_{n, v}$ and $\phi_{n, v^{\prime}}$ by some automorphisms of $\mathbb{S}_{v}$ and $\mathbb{S}_{v^{\prime}}$ converging to the identity when $n \rightarrow \infty$ (so we don't change the limit of $\phi_{n, v^{\prime}} \circ \phi_{n, v}^{-1}$ ), we can suppose that for all $n$,

$$
\xi_{1, n}=\xi_{1}, \xi_{2, n}=\xi_{2}, \xi_{3, n}=\xi_{3}, \xi_{1, n}^{\prime}=\xi_{1}^{\prime}, \xi_{3, n}^{\prime}=\xi_{3}^{\prime} \text { and } \xi_{4, n}^{\prime}=\xi_{4}^{\prime} .
$$

Now we consider the projective charts $\sigma$ on $\mathbb{S}_{v}$ and $\sigma^{\prime}$ on $\mathbb{S}_{v^{\prime}}$ defined by:

(1) $\sigma\left(\xi_{1}\right)=0, \sigma\left(\xi_{2}\right)=1$ and $\sigma\left(\xi_{3}\right)=\infty$;

(2) $\sigma^{\prime}\left(\xi_{1}^{\prime}\right)=0, \sigma^{\prime}\left(\xi_{4}^{\prime}\right)=1$ and $\sigma^{\prime}\left(\xi_{3}^{\prime}\right)=\infty$.

The Moebius transformation $M_{n}:=\sigma^{\prime} \circ \phi_{n, v^{\prime}} \circ \phi_{n, v}^{-1} \circ \sigma^{-1}$ fixes 0 and $\infty$ and maps $\sigma\left(\xi_{4}\right)$ to 1 . Thus

$$
M_{n}(z)=\frac{z}{\lambda_{n}} \quad \text { with } \quad \sigma\left(\xi_{4, n}\right) \underset{n \rightarrow \infty}{\longrightarrow} \infty .
$$

Consequently, $M_{n}$ converges locally uniformly outside infinity to the constant map equal to zero. Thus, $\phi_{n, v^{\prime}} \circ \phi_{n, v}^{-1}={\sigma^{\prime}}^{-1} \circ M_{n} \circ \sigma$ converges locally uniformly to the constant $\left(\sigma^{\prime}\right)^{-1}(0)=i_{v^{\prime}}(v)$ outside $\sigma^{-1}(\infty)=i_{v}\left(v^{\prime}\right)$.

Remark 3.19. In fact we can prove that $\left.\mathfrak{B}\left(\widehat{\operatorname{Mod}}_{X}\right)=\overline{\mathfrak{B}(\operatorname{Mod}}\right)$. This equality is the content of [FT. A simple independent proof can be found in $[\mathrm{A}$, where the inclusion $\mathfrak{B}\left(\widehat{\operatorname{Mod}}_{X}\right) \subseteq \overline{\mathfrak{B}\left(\operatorname{Mod}_{X}\right)}$ is proven by induction on card $X$, and the reverse inclusion is proven using Lemma 3.18 and Corollary 3.6 .

\section{ISOMORPHISM CLASSES OF COVERS}

4.1. Introduction. In this subsection we define some notions that one can find with more details in A1 and we also prove a useful technical lemma.

The generalization of marked rational maps (defined in the introduction) is the notion of (holomorphic) cover between trees of spheres. A cover $\mathcal{F}: \mathcal{T}^{Y} \rightarrow \mathcal{T}^{Z}$ 
between two trees of spheres marked by $Y$ and $Z$ is the following data:

- a combinatorial tree map $F: T^{Y} \rightarrow T^{Z}$,

- for each internal vertex $v$ of $T^{Y}$ and $w:=F(v)$ of $T^{Z}$, a holomorphic ramified cover $f_{v}: \mathbb{S}_{v} \rightarrow \mathbb{S}_{w}$ that satisfies the following properties:

- the restriction $f_{v}: \mathbb{S}_{v}-Y_{v} \rightarrow \mathbb{S}_{w}-Z_{w}$ is a cover,

$-f_{v} \circ i_{v}=i_{w} \circ F$

- if $e$ is an edge between two internal vertices $v$ and $v^{\prime}$, then the local degree of $f_{v}$ at $i_{v}(e)$ is the same as the local degree of $f_{v^{\prime}}$ at $i_{v^{\prime}}(e)$.

In A1, it is independently proven that a cover $\mathcal{F}$ between trees of spheres is such that $F$ is surjective and there is a global degree, denoted by $\operatorname{deg}(\mathcal{F})$.

We will denote by $\widehat{\mathbf{R a t}}_{\mathbf{F}}$ the set of covers between trees of spheres with portrait $\mathbf{F}=\left(\left.F\right|_{Y},\left.\operatorname{deg}\right|_{Y}\right)$ and $\mathbf{R a t}_{\mathbf{F}}$ the set of covers between two trees that have a unique internal vertex (we respectively talk about covers between trees of spheres marked by $\mathbf{F}$ and of covers between spheres marked by $\mathbf{F}$ ). The set $\mathbf{R a t}_{\mathbf{F}}$ is naturally identified with the space Rat $\mathbf{F}$ defined in the introduction.

\subsection{Isomorphisms of covers between trees.}

Definition 4.1 (Isomorphism between covers). An isomorphism between two covers between trees of spheres $\mathcal{F}_{1}: \mathcal{T}_{1}^{Y} \rightarrow \mathcal{T}_{1}^{Z}$ and $\mathcal{F}_{2}: \mathcal{T}_{2}^{Y} \rightarrow \mathcal{T}_{2}^{Z}$ is a pair of isomorphisms between trees of spheres $\left(\mathcal{M}^{Y}, \mathcal{M}^{Z}\right)$ such that:

- $\mathcal{T}_{1}^{Y} \sim_{\mathcal{M}^{Y}} \mathcal{T}_{2}^{Y}$ and $\mathcal{T}_{1}^{Z} \sim_{\mathcal{M}^{Z}} \mathcal{T}_{2}^{Z}$

- for all the vertices $v_{1} \in T_{1}^{Y}, v_{2}:=M^{Y}\left(v_{1}\right) \in T_{2}^{Y}, w_{1}:=F_{1}\left(v_{1}\right) \in T_{1}^{Z}$ and $w_{2}:=F_{2}\left(v_{2}\right) \in T_{2}^{Z}$, the following diagram commutes:

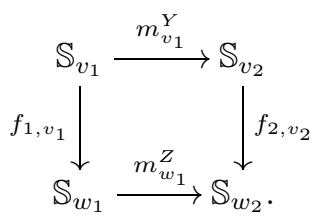

Thus we write $\mathcal{F}_{1} \sim \mathcal{F}_{2}$ or $\mathcal{F}_{1} \sim{ }_{\left(\mathcal{M}^{Y}, \mathcal{M}^{Z}\right)} \mathcal{F}_{2}$. As $\mathcal{M}^{Y}$ and $\mathcal{M}^{Z}$ are invertible, it is an equivalence relation. Equivalence classes of this relation are called isomorphism classes of covers between trees of spheres.

Note that two covers between trees of spheres which are isomorphic have the same degree and same portrait. Thus we can talk about the degree and the portrait of an isomorphism class of covers between trees of spheres.

We denote by $\widehat{\operatorname{rat}}_{\mathbf{F}}$ the quotient of $\widehat{\mathbf{R a t}}_{\mathbf{F}}$ by this equivalence relation and $\mathbf{r a t}_{\mathbf{F}}$ the one of $\mathbf{R a t}_{\mathbf{F}}$.

4.3. Marked covers, projections and topology. Recall that $\mathfrak{T}_{X}$ denotes the set of trees of spheres marked by $X$. Define

$$
\mathcal{I}: \widehat{\operatorname{Rat}}_{\mathbf{F}} \rightarrow \mathfrak{T}_{Y} \times \mathfrak{T}_{Z}
$$

that associates $\left(\mathcal{T}^{Y}, \mathcal{T}^{Z}\right)$ to $\mathcal{F}: \mathcal{T}^{Y} \rightarrow \mathcal{T}^{Z}$. We prove the following proposition by recurrence on the cardinal of $Y$.

Proposition 4.2. The map $\mathcal{I}: \widehat{\operatorname{Rat}}_{\mathbf{F}} \rightarrow \mathfrak{T}_{Y} \times \mathfrak{T}_{Z}$ is an injection. It descends to an injective map in the quotient:

$$
[I]: \widehat{\operatorname{rat}}_{\mathbf{F}} \rightarrow \operatorname{Mod}_{Y} \times \operatorname{Mod}{ }_{Z}
$$


The proof of this proposition follows essentially from the following fact: two maps from the Riemann sphere to itself such that all the preimages of three distinct points coincide (with multiplicity) are equals. First we make the following remark:

Remark 4.3. Every tree of spheres is either a marked sphere or it has an internal vertex which is adjacent to exactly another one. Indeed, in the not a marked sphere case, one can easily check that if $C=\left[v_{1}, v_{2}, v_{3}, \ldots, v_{k-1}, v_{k}\right]$ is a path of maximal cardinality, then $v_{3}$ works. (cf. detailed proof in [A]).

Proof of Proposition 4.2. We prove this result by induction on the cardinal of $Y$.

We begin with the case $\operatorname{card}(Y)=3$. Take $\mathcal{F}: \mathcal{T}^{Y} \rightarrow \mathcal{T}^{Z}$ in $\widehat{\boldsymbol{R a t}}_{\mathbf{F}}$. We prove that $\mathcal{F}$ is uniquely determined by $\mathcal{I}(\mathcal{F})$. If $Y$ has only three elements, then $T^{Y}$ has a unique internal vertex $v$. Then $T^{Z}$ has only one internal vertex $v^{\prime}$ which is the image of $v$. The combinatorial tree map is uniquely determined. Moreover, as $Z$ has three elements and as we know all their preimages, we know the preimages of three attaching points of three edges on $\mathbb{S}_{v^{\prime}}$ by $f_{v}$. So $f_{v}$ is also uniquely determined.

Let $Y$ be a set of cardinal $n>3$. Take $\mathcal{F}: \mathcal{T}^{Y} \rightarrow \mathcal{T}^{Z}$ in $\widehat{\mathbf{R a t}}_{\mathbf{F}}$. Suppose that we know $\left(\mathcal{T}^{Y}, \mathcal{T}^{Z}\right)$ and we prove that $\mathcal{F}$ is uniquely determined.

If $T^{Y}$ has only one internal vertex, then we do the same proof as before. We suppose that it is not the case. According to Remark 4.3, $T^{Y}$ has an internal vertex $v$ adjacent to a unique internal vertex. Let $y$ be a leaf adjacent to $v$ (it exists because $T^{Y}$ is stable). The image of $v$ is necessarily adjacent to $z:=F(y)$ which is a leaf. Hence $w:=F(v)$ is uniquely determined. As $T^{Y}$ has more than one internal vertex, $w$ is adjacent to an internal vertex $w^{\prime}$. The preimages of $w$ are the vertices adjacent to the preimages of the $z$. Similarly the preimages of $w^{\prime}$ are all the internal vertices adjacent to $v$. Thus the preimages of $w$ and $w^{\prime}$ are uniquely determined.

Now suppose that $v$ is a preimage of $w$. Given that $T^{Z}$ is stable, $w$ is adjacent to some $z^{\prime \prime} \in Z-\{z\}$. So we know the preimages of two of its points by $f_{v}$. Define $e:=\left\{w, w^{\prime}\right\}$. As we know the preimages of $w^{\prime}$ and of $w$, the preimages by $f_{v}$ of the attaching point of $e$ on $w$ are the attaching points on $v$ of the edges of $v$ connecting $v$ to some internal vertices. As we know the preimages of three distinct points of $w$ by $f_{v}$, the map $f_{v}$ is uniquely determined.

Thus the preimage by $F$ of $B:=B_{w^{\prime}}(e)$ is uniquely determined. Define $T^{\prime \prime}:=$ $V^{Z} \backslash B$ and $T^{\prime}:=T^{Y} \backslash F^{-1}(B)=F^{-1}\left(T^{\prime \prime}\right)$. Now we prove that $\left.F\right|_{T^{\prime}}$ is uniquely determined.

Let $E \subset T^{Z}$ be the set of the leaves adjacent to $w$ and of the edges adjacent to them. The graph $T^{\prime \prime}:=T^{Z} \backslash E$ is clearly a tree and we know the preimages of its leaves by $F$. Let $T^{\prime}$ be any connected component of $T^{Y}$ minus the preimages of $E$. This is a tree whose leaves are the elements of $Y \backslash F^{-1}(E \cap Z)$ and the preimages of $w^{\prime}$, thus we know the map $F$ on its set of leaves. We define $\mathcal{T}^{\prime \prime}$ to be the tree of spheres of combinatorial tree $T^{\prime}$ and for which the spheres associated to the internal vertices and the attaching points of edges are the same as the one for $\mathcal{T}^{Y}$. Similarly we define $\mathcal{T}^{\prime \prime}$ from $T^{\prime}$ and $\mathcal{T}^{Z}$.

It is easy to check that the natural restriction $\mathcal{F}^{\prime}: \mathcal{T}^{\prime} \rightarrow \mathcal{T}^{\prime \prime}$ of $\mathcal{F}$ is a cover between trees of spheres. As the set of leaves of $T^{\prime \prime}$ has less elements than the one of $T^{Y}$ and as we know the portrait of $\mathcal{F}^{\prime}$, the induction allows one to reconstruct $\mathcal{F}: \mathcal{T}^{\prime} \rightarrow \mathcal{T}^{\prime \prime}$ from the pair $\left(\mathcal{T}^{\prime}, \mathcal{T}^{\prime \prime}\right)$.

Denote by $\pi_{1}$ the projection on the first coordinate. 
Definition 4.4. We define the map

$$
\mathbf{I}: \widehat{\operatorname{rat}}_{\mathbf{F}} \rightarrow \widehat{\operatorname{Mod}}_{Y} \text { by setting } \mathbf{I}:=\pi_{1} \circ[I] .
$$

Proposition 4.5. The map $\mathbf{I}: \widehat{\operatorname{rat}}_{\mathbf{F}} \rightarrow \widehat{\operatorname{Mod}}_{Y}$ is injective.

Proof. Take $\mathcal{F}: \mathcal{T}^{Y} \rightarrow \mathcal{T}^{Z}$ in $\widehat{\mathbf{R a t}}_{\mathbf{F}}$. Let $v_{0}$ be a vertex given by Remark 4.3, Let $v_{0}^{\prime}$ be its image. Let $V_{0}$ be the set of the leaves adjacent to $v_{0}$. The portrait $(F, \operatorname{deg})$ determines the images of the elements of $V_{0}$ that have to be adjacent to $v_{0}^{\prime}$. The other preimages of $v_{0}$ are adjacent to the elements of $F^{-1} \circ F\left(V_{0}\right)$. As we did in the proof of Proposition 4.2, we determine the vertices of the tree $T^{Z}$ and the map $F$ from the data of $T^{Y} \backslash F^{-1}\left(v_{0}^{\prime}\right)$ by induction on the number of vertices of $T^{Y}$.

Thus, it is possible to reconstruct the combinatorial tree $T^{Z}$ and the combinatorial tree map from $\mathcal{T}^{Y}$. We prove that the attaching points of the edges of $T^{Z}$ on the vertices of $\mathcal{T}^{Z}$ are well determined up to post-composition by automorphisms. For this, it is sufficient to show that for each internal vertex $v$ of $T^{Z}$, the attaching points of $E_{v}$ are determined by the data of three of them.

For every internal vertex $v$ of $T^{Z}$, we suppose that we know the attaching points $z_{0}, z_{1}$ and $z_{\infty}$ of three distinct edges $e_{0}, e_{1}, e_{\infty}$ on $v$. For every preimage $w$ of $v$, there exists a unique holomorphic cover $f_{w}: \mathbb{S}_{w} \rightarrow \mathbb{S}_{v}$ mapping the preimages of the edge $e_{0}$ (resp. $\left.e_{1}, e_{\infty}\right)$ on $z_{0}$ (resp. $\left.z_{1}, z_{\infty}\right)$. If $e$ is an edge on $v$, then $e$ has a preimage $e^{\prime}$ on $w$ so its attaching point has to be $f_{w}\left(e_{w}^{\prime}\right)$.

We define a topology on the set of isomorphism classes of the covers between trees of spheres via the map $\mathbf{I}$.

4.4. Convergence notion. We define the notion of convergence of a sequence of marked spheres covers to marked cover between trees of spheres as follows.

Definition 4.6 (Non-dynamical convergence). A sequence of

$$
\mathcal{F}_{n}:=\left(f_{n}, a_{n}^{Y}, a_{n}^{Z}\right) \in \mathbf{R a t}_{\mathbf{F}}
$$

converges to $\mathcal{F}: \mathcal{T}^{Y} \rightarrow \mathcal{T}^{Z}$ in $\widehat{\mathbf{R a t}}_{\mathbf{F}}$ if and only if for all pair of internal vertices $v$ and $w:=F(v)$, there exists sequences of isomorphisms $\phi_{n, v}^{Y}: \mathbb{S}_{n}^{Y} \rightarrow \mathbb{S}_{v}$ and $\phi_{n, w}^{Z}: \mathbb{S}_{n}^{Z} \rightarrow \mathbb{S}_{w}$ such that

- $\phi_{n, v}^{Y} \circ a_{n}^{Y}: Y \rightarrow \mathbb{S}_{v}$ converges to $a_{v}^{Y}: Y \rightarrow \mathbb{S}_{v}$,

- $\phi_{n, w}^{Z} \circ a_{n}^{Z}: Z \rightarrow \mathbb{S}_{w}$ converges to $a_{w}^{Z}: Z \rightarrow \mathbb{S}_{w}$ and

- $\phi_{n, w}^{Z} \circ f_{n} \circ\left(\phi_{n, v}^{Y}\right)^{-1}: \mathbb{S}_{v} \rightarrow \mathbb{S}_{w}$ converges locally uniformly outside $Y_{v}$ to $f_{v}: \mathbb{S}_{v} \rightarrow \mathbb{S}_{w}$

We use the notation $\mathcal{F}_{n} \rightarrow \mathcal{F}$ or $\mathcal{F}_{n} \underset{\left(\phi_{n}^{Y}, \phi_{n}^{Z}\right)}{\longrightarrow} \mathcal{F}$.

Let us prove that this topology is compatible with the convergence notion.

Lemma 4.7. Let $\left(f_{n}\right)_{n}$ and $\left(f_{n}^{\prime}\right)_{n}$ be two sequences in $\mathbf{R a t}_{\mathbf{F}}$. For $\mathcal{F}$ and $\mathcal{F}^{\prime}$ in $\widehat{\operatorname{Rat}}_{\mathbf{F}}$ we have:

(1) (quotient)

- if $\mathcal{F} \sim \mathcal{F}^{\prime}$, then $f_{n} \rightarrow \mathcal{F} \Longleftrightarrow f_{n} \rightarrow \mathcal{F}^{\prime}$,

- if $f_{n} \sim f_{n}^{\prime}$, then $f_{n} \rightarrow \mathcal{F} \Longleftrightarrow f^{\prime}{ }_{n} \rightarrow \mathcal{F}$.

(2) (unicity of the limit) if $f_{n} \rightarrow \mathcal{F}$ and $f_{n} \rightarrow \mathcal{F}^{\prime}$, then $\mathcal{F} \sim \mathcal{F}^{\prime}$. 
Proof. If $f_{n} \underset{\left(\phi_{n}^{Y}, \phi_{n}^{Z}\right)}{\longrightarrow} \mathcal{F}$ and $\mathcal{F} \sim_{\left(\mathcal{M}^{Y}, \mathcal{M}^{Z}\right)} \mathcal{F}^{\prime}$, then

$$
f_{n} \underset{\left(\psi_{n}^{Y}, \psi_{n}^{Z}\right)}{\longrightarrow} \mathcal{F}^{\prime} \text { with } \psi_{n, v}^{\star}:=M_{v}^{\star} \circ \phi_{n, v}^{\star} .
$$

Moreover, suppose that $f_{n}^{\prime} \sim_{\left(\mathcal{M}_{n}^{Y}, \mathcal{M}_{n}^{Z}\right)} f_{n}$,

$$
\text { if } f_{n} \underset{\left(\phi_{n}^{Y}, \phi_{n}^{Z}\right)}{\longrightarrow} \mathcal{F} \text {, then } f_{n}^{\prime} \underset{\left(\mathcal{M}^{Y} \circ \phi_{n}^{\left.Y, \mathcal{M}^{Y} \circ \phi_{n}^{Z}\right)}\right.}{\longrightarrow} \mathcal{F} \text {. }
$$

So this convergence notion is well behaved under the quotient.

To prove (2), we suppose that $f_{n} \underset{\left(\phi_{n}^{Y}, \phi_{n}^{Z}\right)}{\longrightarrow} \mathcal{F}$ and $f_{n} \underset{\left(\psi_{n}^{Y}, \psi_{n}^{Z}\right)}{\longrightarrow} \mathcal{F}^{\prime}$. Then

$$
\mathcal{F} \sim_{\left(\mathcal{M}^{Y}, \mathcal{M}^{Z}\right)} \mathcal{F}^{\prime} \text { with } m_{v}^{\star}:=\lim _{n \rightarrow \infty} \psi_{n, v}^{\star} \circ\left(\phi_{n, v}^{\star}\right)^{-1} .
$$

Indeed, $\left(a_{n, v}^{\star}\right)_{n}$ converges to $a_{v}^{\star}$ and $\left(\left(\psi_{n, v}^{\star} \circ\left(\phi_{n, v}^{\star}\right)^{-1}\right)^{\star} \circ a_{n, v}^{\star}\right)_{n}$ converges to $a_{v}^{\prime \star}$ on $Y$ which contains at least three points, so $m_{v}$ is an isomorphism.

Corollary 4.8. The convergence notion defined on $\widehat{\mathbf{R a t}}_{\mathbf{F}}$ implies the one given by the topology given by $\mathbf{I}$ :

$$
\text { if } f_{n} \rightarrow \mathcal{F} \text {, then } \mathbf{I}\left(\left[f_{n}\right]\right) \rightarrow \mathbf{I}([\mathcal{F}]) \text {. }
$$

Proof. Indeed, if $\left(f_{n}: \mathcal{T}_{n}^{Y} \rightarrow \mathcal{T}_{n}^{Z}\right) \rightarrow\left(\mathcal{F}: \mathcal{T}^{Y} \rightarrow \mathcal{T}^{Z}\right)$, then by definition we have $\mathcal{T}_{n}^{Y} \rightarrow \mathcal{T}^{Y}$, i.e., $I\left(f_{n}\right) \rightarrow I(\mathcal{F})$ so in the quotient $\mathbf{I}\left(\left[f_{n}\right]\right) \rightarrow \mathbf{I}([\mathcal{F}])$.

We will prove the reciprocal property in the next section.

Here is a nice property to note:

Lemma 4.9. Let $\mathcal{F}: \mathcal{T}^{Y} \rightarrow \mathcal{T}^{Z}$ be in $\widehat{\mathbf{R a t}}_{\mathbf{F}}$. Let $v$ be an internal vertex of $T^{Y}$ with $\operatorname{deg}(v)=\operatorname{deg} \mathcal{F}$ and let $\mathcal{F}_{n}:=\left(f_{n}, a_{n}^{Y}, a_{n}^{Z}\right) \in \boldsymbol{R a t}_{\mathbf{F}}$ such that $\mathcal{F}_{n} \underset{\phi_{n}^{Y}, \phi_{n}^{Z}}{\longrightarrow} \mathcal{F}$. Then the sequence $\phi_{n, F(v)}^{Z} \circ f_{n} \circ\left(\phi_{n, v}^{Y}\right)^{-1}: \mathbb{S}_{v} \rightarrow \mathbb{S}_{F(v)}$ converges uniformly to $f_{v}: \mathbb{S}_{v} \rightarrow \mathbb{S}_{F(v)}$.

This property is not used in this paper but the interested reader can find a proof in $\mathrm{A} 1$.

4.5. Compactness. In this section we prove

$$
\mathbf{I}\left(\widehat{\operatorname{rat}}_{\mathbf{F}}\right)=\overline{\mathbf{I}\left(\operatorname{rat}_{\mathbf{F}}\right)} \text {. }
$$

We end the section by the proof of Theorem 1.

We first prove the fundamental result:

Lemma 4.10. Let $\left(f_{n}: \mathbb{S} \rightarrow \mathbb{S}\right)_{n}$ be a sequence of rational maps of same degree. Then, there exists a subsequence $\left(f_{n_{k}}\right)_{n_{k}}$ and a sequence of Moebius transformations $\left(M_{n_{k}}\right)_{n_{k}}$ such that $\left(M_{n_{k}} \circ f_{n_{k}}\right)_{n_{k}}$ converges to a non-constant rational map $f$ uniformly outside a finite number of points.

Proof. Define $x_{0}=\infty$. We extract a subsequence in order to have

$$
X_{n}:=f_{n}^{-1} \circ f_{n}\left(x_{0}\right) \rightarrow X
$$

with multiplicity. Choose $y_{0} \in \mathbb{C} \backslash X$. We extract a subsequence in order to have

$$
Y_{n}:=f_{n}^{-1}\left(f_{n}\left(y_{0}\right)\right) \rightarrow Y
$$

with multiplicity. Choose $z_{0} \in \mathbb{C} \backslash(X \cap Y)$. Again, we extract a subsequence in order to have

$$
Z_{n}:=f_{n}^{-1}\left(f_{n}\left(z_{0}\right)\right) \rightarrow Z
$$


By construction, for all $n$ we can find a Moebius transformation satisfying:

$$
M_{n} \circ f_{n}\left(x_{0}\right)=\infty, M_{n} \circ f_{n}\left(y_{0}\right)=0, M_{n} \circ f_{n}\left(z_{0}\right)=1 .
$$

Thus we have

$$
\forall w \in \mathbb{C}, M_{n} \circ f_{n}(w)=\frac{\prod_{x \in X_{n}}(w-x)}{\prod_{y \in Y_{n}}(w-y)} \cdot \frac{\prod_{y \in Y_{n}}\left(z_{0}-y\right)}{\prod_{x \in X_{n}}\left(z_{0}-x\right)} .
$$

This sequence of rational maps converges uniformly to a non-constant rational map outside a finite number of points which correspond to $X \cap Y$.

Proposition 4.11. Let $\left(\mathcal{F}_{n}\right)_{n}$ be a sequence in $\mathbf{R a t}_{\mathbf{F}}$. If $\left(\mathbf{I}\left(\left[\mathcal{F}_{n}\right]\right)\right)_{n}$ converges, then there exists a cover between trees of spheres $\mathcal{F}$ such that $\left(\mathcal{F}_{n}\right)_{n}$ converges to $\mathcal{F}$.

Proof. Let $\left(\mathcal{F}_{n}: \mathcal{T}_{n}^{Y} \rightarrow \mathcal{T}_{n}^{Z}\right)_{n}$ be a sequence of element of $\mathbf{R a t}_{\mathbf{F}}$ and $\mathcal{T}^{Y} \in \mathfrak{T}_{Y}$ such that $\left(\mathbf{I}\left(\left[\mathcal{F}_{n}\right)\right]\right)_{n}$ converges to $\mathcal{T}^{Y}$. Let $\phi_{n}^{Y}$ be such that

$$
\mathcal{T}_{n}^{Y} \rightarrow_{\phi_{n}^{Y}} \mathcal{T}^{Y} \in \mathfrak{T}_{Y}
$$

Fix some charts $\sigma_{n}^{Z}: \mathbb{S}_{n}^{Z} \rightarrow \mathbb{S}$. For every internal vertex $v$ of $\mathcal{T}^{Y}$, we set $\tilde{f}_{n, v}:=\sigma_{n}^{Z} \circ f_{n} \circ\left(\phi_{n, v}^{Y}\right)^{-1}$. According to Lemma 4.10, after passing to a subsequence, we can find a sequence of isomorphisms $\left(M_{n, v}: \mathbb{S} \rightarrow \mathbb{S}\right)_{n}$ such that $\left(M_{n, v} \circ \tilde{f}_{n, v}\right)_{n}$ converges uniformly outside a finite number of points to a non-constant holomorphic morphism $\tilde{f}_{v}: \mathbb{S}_{v} \rightarrow \mathbb{S}$. Using compactness of $\widehat{\mathbf{M o d}}_{Z}$, we also take a subsequence so that $\mathcal{T}_{n}^{Z}$ converges to a tree of spheres $\mathcal{T}^{Z} \in \mathfrak{T}_{Z}$. Let $\phi_{n}^{Z}$ be such that

$$
\mathcal{T}_{n}^{Z} \rightarrow_{\phi_{n}^{Z}} \mathcal{T}^{Y} \in \mathfrak{T}_{Z}
$$

We will construct a cover $\mathcal{F}: \mathcal{T}^{Y} \rightarrow \mathcal{T}^{Z}$ that depends only on $\mathcal{T}^{Y}$ and $\mathcal{T}^{Z}$. It follows from Proposition 4.5 that $\mathcal{T}^{Z}$ is unique. Hence, passing here to a subsequence does not affect the result. We set

- $\sigma_{n, v}^{Z}:=M_{n, v} \circ \sigma_{n}^{Z}$

- $\tilde{a}_{v}:=\lim _{n \rightarrow \infty} \sigma_{n, v}^{Z} \circ a_{n}^{Z}$;

- $Y_{v}:=a_{v}(Y)$ and $\tilde{Z}_{v}:=\tilde{a}_{v}(Z)$.

Note that $\tilde{f}_{v}\left(Y_{v}\right)=\tilde{a}_{v}(Z)$.

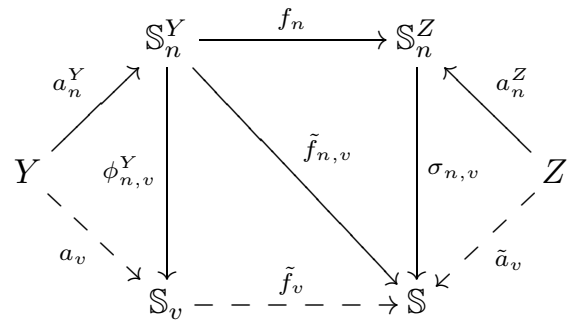

Claim 1. Let $\gamma_{z}$ be the boundary of a small disk around $z \in \tilde{Z}_{v}$. Let $y \in Y_{v}$ be such that $\tilde{f}_{v}(y)=z$. Then there exists $\gamma_{y}$ surrounding $y$ such that $\tilde{f}_{v}\left(\gamma_{y}\right)=\gamma_{z}$ and $\tilde{f}_{n, v}\left(\gamma_{y}\right) \rightarrow \gamma_{z}$.

Proof. Indeed, if $\gamma_{z}$ is small enough, $\tilde{f}_{v}^{-1}\left(\gamma_{z}\right)$ is a loop $\gamma_{y}$ which is the boundary of a disk containing $y$ and avoiding the other elements of $Y_{v}$. As on $\mathbb{S}_{v} \backslash Y_{v}$, the convergence is uniform, so $\tilde{f}_{n, v}\left(\gamma_{y}\right) \rightarrow \gamma_{z}$.

Claim 2. For every internal vertex $v$ of $\mathcal{T}^{Y}$, we have $\operatorname{card} \tilde{Z}_{v} \geq 3$. 
Proof. Consider small disks around the elements of $\tilde{Z}_{v}$. Suppose $n$ large enough such that the $\tilde{f}_{n, v}(Y)$ are in these disks. Denote by $D_{Z}$ the set $\mathbb{S}$ minus these disks and $D_{Y}:=\tilde{f}_{v}^{-1}\left(D_{Z}\right)$. The Riemann-Hurwitz formula gives

$$
-1 \geq \chi\left(D_{Y}\right)=\operatorname{deg}\left(\tilde{f}_{v}\right) \chi\left(D_{Z}\right)
$$

because $\mathbb{S}_{v}$ has at least three edges and $D_{Y}$ has no critical points. $\operatorname{As} \operatorname{deg}\left(\tilde{f}_{v}\right) \geq 1$, then $\chi\left(D_{Z}\right) \leq-1$ so $\operatorname{card} \tilde{Z}_{v} \geq 3$.

Thus card $\left(\tilde{a}_{v}(Z)\right) \geq 3$. Let $t_{v^{\prime}}$ be a triple of points of $Z$ which have pairwise distinct images by $\tilde{a}_{v}$. Let $v^{\prime}$ be the unique vertex of $T^{Z}$ separating $t_{v^{\prime}}$. As on the diagram below we use the notation $\sigma_{n, v^{\prime}}:=\phi_{n, v^{\prime}}^{Z} \circ \sigma_{n, v}^{-1}$.

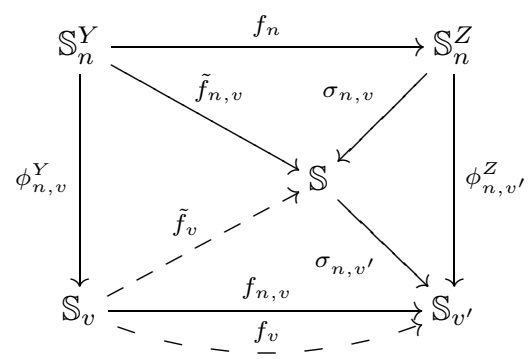

From the choice of $t_{v^{\prime}}$, we know that $\sigma_{n, v^{\prime}}$ converges to an isomorphism $\sigma_{v^{\prime}}$. Thus $\sigma_{n, v^{\prime}} \circ \tilde{f}_{n, v} \rightarrow \sigma_{v^{\prime}} \circ \tilde{f}_{v}:=f_{v}$ locally uniformly outside a finite number of points and $\operatorname{deg}\left(f_{v}\right) \geq 1$.

So we have $f_{n, v}:=\phi_{n, v^{\prime}}^{Z} \circ f_{n} \circ\left(\phi_{n, v}^{Y}\right)^{-1} \rightarrow f_{v}$ locally uniformly outside a finite number of points and $\operatorname{deg}\left(f_{v}\right) \geq 1$.

Let $I V^{Y}$ and $I V^{Z}$ denote the respective sets of internal vertices of $\mathcal{T}^{Y}$ and $\mathcal{T}^{Z}$.

Claim 3. The map $F: I V^{Y} \rightarrow I V^{Z}$ that maps $v$ defined by $t_{v}$ to the vertex defined by $t_{v^{\prime}}$ extends to a map between trees.

Proof. Let $v_{1}$ and $v_{2}$ be two adjacent vertices in $T^{Y}$ connected by an edge $e$ and let $v_{1}^{\prime}$ and $v_{2}^{\prime}$ be their respective images. Let $D_{1}$ (resp. $D_{2}$ ) be a topological disk neighborhood of the attaching point of $e$ on $v_{1}$ (resp. $v_{2}$ ) and containing only this attaching point of edge and let $C_{1}$ (resp. $C_{2}$ ) be its boundary. Denote by $A_{n}:=\left(\phi_{n, v_{1}}^{Y}\right)^{-1}\left(D_{1}\right) \cap\left(\phi_{n, v_{2}}^{Y}\right)^{-1}\left(D_{2}\right) \subset \mathbb{S}_{n}$. Denote by $C_{\star}^{\prime}:=f_{v_{\star}}\left(C_{\star}\right)$ and $A_{n}^{\prime}:=f_{n}\left(A_{n}\right)$. We now suppose that $n$ is large enough such that $A_{n}$ is an annulus and does not contain any attaching point of edges. Thus $A_{n}^{\prime}$ does not contain any attaching point of edges either. As the critical points of $f_{n}$ are attaching points of edges, $A_{n}$ does not contain critical points and $A_{n}^{\prime}$ is an annulus.

Suppose that there is a vertex $v^{\prime}$ between $v_{1}^{\prime}$ and $v_{2}^{\prime}$. As $\phi_{n, v_{\star}}^{Z}\left(C_{\star}^{\prime}\right) \rightarrow C_{\star}^{\prime}$, Lemma 3.18 implies that $\phi_{n, v^{\prime}}^{Z}\left(A_{n}^{\prime}\right)$ tends to $\mathbb{S}_{v^{\prime}}$ minus the attaching points of the branches containing respectively $v_{1}^{\prime}$ and $v_{2}^{\prime}$. As $A_{n}^{\prime}$ does not contain attaching points of edge, $\mathbb{S}_{v^{\prime}}$ has only two attaching points of edges which contradicts the stability of $T^{Z}$. Thus $F$ maps two adjacent vertices to two adjacent vertices.

In particular we proved that the image of the attaching point of $e$ on $v_{\star}$ is the attaching point of $F(e)$ on $v_{\star}^{\prime}$ i.e., $f_{v_{\star}}\left(e_{v_{\star}}\right)$.

Claim 4. The map $\mathcal{F}: \mathcal{T}^{Y} \rightarrow \mathcal{T}^{Z}$ defined by $F$ and the $f_{v}$ is a cover between trees of spheres. 
Proof. Take $v_{1}^{\prime}:=F\left(v_{1}\right)$ with $v_{1}$ an internal vertex of $T^{Y}$. Let $e^{\prime}:=\left\{v_{1}^{\prime}, v_{2}^{\prime}\right\}$ be an edge of $T^{Z}$. Let $C_{1}^{\prime}$ (resp. $C_{2}^{\prime}$ ) be a topological circle surrounding a disk $D_{1}^{\prime}$ (resp. $D_{2}^{\prime}$ ) containing a unique attaching point on $v_{1}^{\prime}$ (resp. on $v_{2}^{\prime}$ ), the one of $e^{\prime}$. Define $A_{n}^{\prime}=\phi_{n, v_{1}^{\prime}}^{Z}\left(D_{1}^{\prime}\right) \cap \phi_{n, v_{2}^{\prime}}^{Z}\left(D_{2}^{\prime}\right)$ and suppose $n$ large enough such that $A_{n}^{\prime}$ is an annulus. Let $A_{n}$ be a connected component of $f_{n}^{-1}\left(A_{n}^{\prime}\right)$. From the RiemannHurwitz formula, we deduce that $A_{n}$ is an annulus. Denote by $C_{1, n}$ and $C_{2, n}$ the preimages of $C_{1}^{\prime}$ and $C_{2}^{\prime}$ surrounding $A_{n}$ and by $D_{1, n}$ the disks bounded by $C_{1, n}$ containing $A_{n}$. We suppose $n$ large enough such that the partition of $a_{n}(Y)$ (resp. $\left.a_{n}(Z)\right)$ given by the two connected components of $\mathbb{S}_{n} \backslash A_{n}$ (resp. $\mathbb{S}_{n} \backslash A_{n}^{\prime}$ ) is constant.

Take $z_{2} \in Z \cap B_{v_{1}^{\prime}}(F(e))$ and $z_{1} \in Z \cap B_{v_{2}^{\prime}}(F(e))$. Then $a_{n}\left(z_{1}\right)$ and $a_{n}\left(z_{2}\right)$ are respectively in each of the two connected components of $\mathbb{S}_{n} \backslash A_{n}^{\prime}$. After choosing a projective chart $\sigma_{n}$ such that $\sigma_{n} \circ a_{n}\left(z_{1}\right)=0$ and $\sigma_{n} \circ a_{n}\left(z_{2}\right)=\infty$, we suppose that $\mathbb{S}_{n}=\mathbb{S}, a_{n}\left(z_{1}\right)=0$ and $a_{n}\left(z_{2}\right)=\infty$.

Denote by

$$
n_{0}:=\operatorname{card}\left\{y \in Y \cap D_{1, n} \mid f_{n}(y)=0\right\}
$$

and

$$
n_{\infty}:=\operatorname{card}\left\{y \in Y \cap D_{1, n} \mid f_{n}(y)=\infty\right\} .
$$

The local degree of $f_{v_{1}}$ at the attaching point of $e$ is the same as the degree of $f_{v_{1}}$ on $C_{1, n}$ which is the one of $f_{n}$ on $\left(\phi_{n, v_{1}}^{Y}\right)^{-1}\left(C_{1, n}\right)$, i.e.,

$$
\operatorname{deg}_{f_{v_{1}}}(e)=n_{0}-n_{\infty} .
$$

Note that these two cardinals don't depend on the choice of the pair $\left(z_{1}, z_{2}\right)$ in the connected components of $\mathbb{S}_{n} \backslash A_{n}^{\prime}$. Again these cardinals are the same if we consider $D_{2}$ instead of $D_{1}$ because $A_{n}$ does not contain critical values. By the same deductions on $v_{2}$ we prove that $\operatorname{deg}_{f_{v_{1}}}(e)=\operatorname{deg}_{f_{v_{2}}}(e)$.

In particular, if $n_{0} \neq 0$, then $\phi_{n, v_{1}}\left(D_{1, n}\right)$ contains an attaching point of an edge; thus every preimage of an edge attaching point is the attaching point of an edge. As the image of an edge attaching point is an edge attaching point, $f_{v}: Y_{v} \rightarrow Z_{F(v)}$ is a cover. Moreover the critical points of $f_{v}$ are the limits of the critical points of $\phi_{n, F(v)}^{Z} \circ f_{n} \circ\left(\phi_{n, v}^{Y}\right)^{-1}$ so they are attaching points of edges.

This concludes the proof of Proposition 4.11 because as required we have

$$
\mathcal{F}_{n} \underset{\left(\phi_{n}^{Y}, \phi_{n}^{Z}\right)}{\longrightarrow} \mathcal{F} \text {. }
$$

Corollary 4.12. The topology given by $\mathbf{I}$ is compatible with the convergence notion defined on $\widehat{\mathbf{R a t}}_{\mathbf{F}}$ :

$$
\mathcal{F}_{n} \rightarrow \mathcal{F} \quad \text { if and only if } \quad \mathbf{I}\left(\left[\mathcal{F}_{n}\right]\right) \rightarrow \mathbf{I}([\mathcal{F}]) .
$$

Proof. The implication is given by Corollary 4.8, Reciprocally if

$$
\mathbf{I}\left(\left[\mathcal{F}_{n}: \mathcal{T}_{n}^{Y} \rightarrow \mathcal{T}_{n}^{Z}\right]\right) \rightarrow \mathbf{I}\left(\left[\mathcal{F}: \mathcal{T}^{Y} \rightarrow \mathcal{T}^{Z}\right]\right),
$$

then according to Proposition 4.11, $\left(\mathcal{F}_{n}\right)_{n}$ converges to a cover between trees of spheres $\mathcal{F}^{\prime}$ so $\mathbf{I}\left(\left[\mathcal{F}_{n}\right]\right) \rightarrow \mathbf{I}\left(\left[\mathcal{F}^{\prime}\right]\right)$. We deduce that $\mathbf{I}\left(\left[\mathcal{F}^{\prime}\right]\right)=\mathbf{I}([\mathcal{F}])$, thus $\mathcal{F}=\mathcal{F}^{\prime}$ according to Proposition 4.5 .

We can also directly deduce the theorem assumed in A1. 
Corollary 4.13. Let $y_{n}$ and $z_{n}$ be two sequences of spheres marked respectively by the finite sets $Y$ and $Z$ each one containing at least three elements and converging to the trees of spheres $\mathcal{T}^{Y}$ and $\mathcal{T}^{Z}$.

Every sequence of marked spheres covers $\left(f_{n}, y_{n}, z_{n}\right)_{n}$ of a given portrait converges to a cover between the trees of spheres $\mathcal{T}^{Y}$ and $\mathcal{T}^{Z}$.

Hence we have proven:

Proposition 4.14. The set $\mathbf{I}\left(\widehat{\operatorname{rat}}_{\mathbf{F}}\right)$ is closed. In particular

$$
\overline{\mathbf{I}\left(\operatorname{rat}_{\mathbf{F}}\right)} \subseteq \mathbf{I}\left(\widehat{\operatorname{rat}}_{\mathbf{F}}\right)
$$

Let us now prove the reverse inclusion.

Proposition 4.15. The set $\mathbf{R a t}_{\mathbf{F}}$ is dense in $\widehat{\mathbf{R a t}}_{\mathbf{F}}$. In particular we have

$$
\mathbf{I}\left(\widehat{\operatorname{rat}}_{\mathbf{F}}\right) \subseteq \overline{\mathbf{I}\left(\operatorname{rat}_{\mathbf{F}}\right)} \text {. }
$$

Proof. Take $\mathcal{F}: \mathcal{T}^{Y} \rightarrow \mathcal{T}^{Z}$ in $\widehat{\mathbf{R a t}}_{\mathbf{F}}$. In this proof, we don't distinguish the spheres at the vertices of $\mathcal{T}^{Y}$ and $\mathcal{T}^{Z}$ and some of their projective charts arbitrarily chosen. Take $1>\varepsilon>0$. Take an edge $e$ between two vertices $v_{1}, v_{2}$. Define by $v_{i}^{\prime}:=F\left(v_{i}\right)$ and denote by $e^{\prime}:=F(e)$ the edge between $v_{1}^{\prime}$ and $v_{2}^{\prime}$.

Let $A_{1}^{\prime}$ (resp. $A_{2}^{\prime}$ ) be an annulus between the circles of radii $\varepsilon^{2}$ and $\varepsilon$ centered on the attaching point $e_{v_{1}^{\prime}}^{\prime}$ (resp. $e_{v_{2}^{\prime}}^{\prime}$ ). Let $\phi_{\varepsilon}^{e^{\prime}}: A_{1}^{\prime} \rightarrow A_{2}^{\prime}$ be a biholomorphism that exchanges the borders of the two annuli (maps the circle of radius $\varepsilon^{2}$ on $A_{1}^{\prime}$ to the one of radius $\varepsilon$ on $A_{2}^{\prime}$, reversing the orientation, and reciprocally).

Let $A_{i}$ be the preimage of $A_{i}^{\prime}$ on $v_{i}$. We consider an $\varepsilon$ small enough such that the $A_{i}$ are in neighborhoods of the $e_{v_{i}}$ that map with degree $\operatorname{deg}_{f_{v_{i}}}\left(e_{v_{i}}\right)$ and such that each of these neighborhoods contain a unique edge attaching point. As $\mathcal{F}$ is a covering between trees of spheres, we have $d_{e}:=\operatorname{deg}_{f_{v_{1}}}\left(e_{v_{1}}\right)=\operatorname{deg}_{f_{v_{2}}}\left(e_{v_{2}}\right)$. We choose one of the $d_{e}$ biholomorphisms $\phi_{\varepsilon}^{e}$ that makes the following diagram commuting:

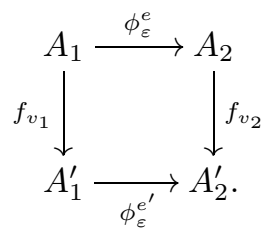

As $F: E^{Y} \rightarrow E^{Z}$ is surjective, after repeating this process we obtain some families $\Phi$ of biholomorphisms associated to the edges between the internal vertices of $T^{Y}$ and $\Phi^{\prime}$ associated to the same one of $T^{Z}$. We suppose $\varepsilon$ small enough such that all the annuli already defined don't have common pairwise intersections. For every internal vertex $v$ of $T^{\star}$, denote by $\mathbb{S}_{\varepsilon, v}^{\star}$ the sphere $\mathbb{S}_{v}^{\star}$ minus some topological closed disks around the attaching points of edges connecting to internal vertices which are bordered by the $A_{i}$ (resp. $A_{i}^{\prime}$ ) as previously defined (but does not contain the $A_{i}\left(\right.$ resp. $\left.\left.A_{i}^{\prime}\right)\right)$. We use the notation

$$
\mathbb{S}_{\varepsilon}^{Y}:=\bigsqcup_{\Phi} \mathbb{S}_{\varepsilon, v}^{Y} \text { and } \mathbb{S}_{\varepsilon}^{Z}:=\bigsqcup_{\Phi^{\prime}} \mathbb{S}_{\varepsilon, v}^{Z}
$$

Every element $y$ of $Y$ is a vertex of $T^{Y}$ which has a unique edge so it is adjacent to a unique internal vertex $v_{y}$ of $T^{Y}$. Denote by $e_{y}$ the attaching point of this edge on $v_{y}$. We define a family of injections $a_{\varepsilon}^{Y}: Y \rightarrow \mathbb{S}_{\varepsilon}^{Y}$ that associate $v_{y}$ to $y$. 
Claim. For $\varepsilon$ small enough, $\mathbb{S}_{\varepsilon}^{Y}$ with $a^{Y}$ is a marked sphere $\mathcal{T}_{\varepsilon}^{Y}$ and if $\varepsilon \rightarrow 0$, we have

$$
\mathcal{T}_{\varepsilon}^{Y} \rightarrow \mathcal{T}^{Y}
$$

Proof. The set of internal vertices of $T^{Y}$ and edges connecting them is a subtree $T^{\prime}$ of $T^{Y}$. Thus it satisfies card $V^{\prime}=\operatorname{card} E^{\prime}+1$ (see for example [Di, Corollary 1.5.3]). In addition the Euler characteristic of $\mathbb{S}_{\varepsilon}^{Y}$ is equal to the sum of the one of the $\widetilde{\mathbb{S}}_{v}^{\varepsilon}$ because the one of an annulus is 0 . But the $\check{\mathbb{S}}_{v}^{\varepsilon}$ are spheres minus a disk for each of the edges of $v \in T^{\prime}$. So the Euler characteristic of $\mathbb{S}_{\varepsilon}^{Y}$ is

$$
\sum\left(2-\operatorname{card} E_{v}^{\prime}\right)=2 \operatorname{card} V^{\prime}-2 \operatorname{card} E^{\prime}=2\left(\operatorname{card} V^{\prime}-\operatorname{card} E^{\prime}\right)=2 .
$$

But $\mathcal{T}^{Y}$ is connected, so $\mathbb{S}_{\varepsilon}^{Y}$ is connected too and it follows that $\mathbb{S}_{\varepsilon}^{Y}$ is a topological sphere. As $\Phi$ is a family of isomorphisms, $\mathbb{S}_{\varepsilon}^{Y}$ is equipped with a complex structure. Thus we proved that $\mathbb{S}_{\varepsilon}^{Y}$ together with $a_{\varepsilon}^{Y}$ is a sphere marked by $Y$ that we will denote by $\mathcal{T}_{\varepsilon}^{Y}$.

Moreover, for every $v$ internal vertex of $T^{Y}$, if we define $\phi_{\varepsilon, v}$ as an isomorphism defined by the identity on $\check{S}_{v}^{\varepsilon}$, then we have $\mathcal{T}_{\varepsilon}^{Y} \rightarrow_{\phi_{\varepsilon}} \mathcal{T}^{Y}$ as required because the $\check{\mathbb{S}} \varepsilon$ tend to the $\mathbb{S}_{v}$.

Similarly we construct a family of injections $a_{\varepsilon}^{Z}: Z \rightarrow \mathbb{S}_{\varepsilon}^{Z}$; then we have the associated trees of spheres $\mathcal{T}_{\varepsilon}^{Z}$ and we have $\mathcal{T}_{\varepsilon}^{Z} \rightarrow \mathcal{T}^{Z}$.

We are now ready to prove that the maps $\mathcal{F}_{\varepsilon}:=\left(\left.\mathcal{F}\right|_{\mathbb{S}_{\varepsilon}^{Y}}: \mathcal{T}_{\varepsilon}^{Y} \rightarrow \mathcal{T}_{\varepsilon}^{Z}\right)$ form a family of covers between marked spheres (for $\varepsilon$ small enough) and $\left[\mathcal{F}_{\varepsilon}\right] \rightarrow[\mathcal{F}]$.

Indeed, for $\varepsilon$ small enough, the $\mathbb{S}_{\varepsilon, v}^{Y}$ for $v$ internal vertex of $T^{Y}$ form a cover of $\mathbb{S}_{\varepsilon}^{Y}$ and the map $\mathcal{F}_{\varepsilon}$ restricted on these is holomorphic; then $f_{\varepsilon}$ is holomorphic. By definition $\left(\left.F_{\varepsilon}\right|_{Y},\left.\operatorname{deg}\right|_{Y}\right)=\mathbf{F}$ so $f_{\varepsilon}$ is a cover on the edges. Thus, for $\varepsilon$ small enough, $\mathcal{F}_{\varepsilon}$ is a cover between marked spheres. In addition we have $\left[\mathcal{T}_{\varepsilon}^{Y}\right] \rightarrow\left[\mathcal{T}^{Y}\right]$.

Proof. (Theorem 1) The equality $\mathbf{I}\left(\widehat{\operatorname{rat}}_{\mathbf{F}}\right)=\overline{\mathbf{I}\left(\mathbf{r a t}_{\mathbf{F}}\right)}$ means that $\widehat{\operatorname{rat}}_{\mathbf{F}}$ is the compact closure of $\operatorname{rat}_{F}$. The injectivity of $\mathbf{I}$ is stated and proven as Proposition 4.5.

\section{DYNAMICS}

5.1. Introduction. In this section we suppose that $X \subseteq Y \cap Z$ is a finite set with at least three elements.

Definition 5.1. Given a finite set $X^{\prime}$, a tree of spheres $\mathcal{T}^{X}$ is compatible with a tree of spheres $\mathcal{T}^{X^{\prime}}$ if $X \subset X^{\prime}$ and for each internal vertex $v$ of $\mathcal{T}^{X}$

- $v$ is also an internal vertex of $\mathcal{T}^{X^{\prime}}$,

- $\mathbb{S}_{v}^{X}=\mathbb{S}_{v}^{X^{\prime}}$, and

- $a_{v}^{X}=\left.a_{v}^{X^{\prime}}\right|_{X}$.

We will say that $\left(\mathcal{F}, \mathcal{T}^{X}\right)$ is a dynamical system between trees of spheres if:

- $\mathcal{F}: \mathcal{T}^{Y} \rightarrow \mathcal{T}^{Z}$ is a cover between trees of spheres,

- $\mathcal{T}^{X}$ is a tree of spheres compatible with $\mathcal{T}^{Y}$ and with $\mathcal{T}^{Z}$.

Dynamical covers between marked spheres can be naturally identified to dynamically marked rational maps:

Recall that a rational map dynamically marked by $(\mathbf{F}, X)$ is a rational map $(f, y, z)$ marked by $\mathbf{F}$ such that $\left.y\right|_{X}=\left.z\right|_{X}$. We denote by $\operatorname{Rat}_{\mathbf{F}, X}$ the set of rational maps dynamically marked by $(\mathbf{F}, X)$ and by $\operatorname{rat}_{\mathbf{F}, X}$ its quotient modulo the action by conjugacy of the group of Moebius transformation. 


\subsection{Isomorphism and topology.}

Definition 5.2. Two dynamical systems between trees of spheres $\left(\mathcal{F}_{1}, \mathcal{T}_{1}^{X}\right)$ and $\left(\mathcal{F}_{2}, \mathcal{T}_{2}^{X}\right)$ are conjugated if there exist two isomorphisms between trees of spheres $\mathcal{M}^{Y}: \mathcal{T}_{1}^{Y} \rightarrow \mathcal{T}_{2}^{Y}$ and $\mathcal{M}^{Z}: \mathcal{T}_{1}^{Z} \rightarrow \mathcal{T}_{2}^{Z}$ such that:

$$
\mathcal{F}_{1} \sim{ }_{\left(\mathcal{M}^{Y}, \mathcal{M}^{Z}\right)} \mathcal{F}_{2}
$$

and for every internal vertex $v \in \mathcal{T}_{1}^{X}$,

$$
m_{v}^{Y}=m_{v}^{Z}
$$

We denote by $\mathbf{D} \mathbf{y} \mathbf{n}_{\mathbf{F}, X}$ the set of such dynamical systems between trees of spheres of portrait $\mathbf{F}$. We denote by $\mathbf{d y n}_{\mathbf{F}, X}$ their conjugacy classes. With this definition the set $\operatorname{rat}_{\mathbf{F}, X}$ defined in the introduction is naturally identified to the set of classes of dynamical systems between marked spheres.

Lemma 5.3. The map that associates to every $\left[\left(\mathcal{F}, \mathcal{T}^{X}\right)\right] \in \mathbf{d y n}_{\mathbf{F}, X}$ the element $[\mathcal{F}] \in \widehat{\mathbf{r a t}}_{\mathbf{F}}$ is an injection.

Proof. Take $\left(\mathcal{F}_{1}, \mathcal{T}_{1}^{X}\right)$ and $\left(\mathcal{F}_{2}, \mathcal{T}_{2}^{X}\right)$ in $\mathbf{D y n}_{\mathbf{F}, X}$ such that $\mathcal{F}_{1} \sim_{\left(\mathcal{M}^{Y}, \mathcal{M}^{Z}\right)} \mathcal{F}_{2}$. We want to prove that $\left[\left(\mathcal{F}_{1}, \mathcal{T}_{1}^{X}\right)\right]=\left[\left(\mathcal{F}_{2}, \mathcal{T}_{2}^{X}\right)\right]$.

It is clear that $\left.M^{Y}\right|_{T_{1}^{X}}=\left.M^{Z}\right|_{T_{1}^{X}}$. Take $v$ as an internal vertex of $T_{1}^{X}$. As $\left(\mathcal{F}_{2}, \mathcal{T}_{2}^{X}\right) \in \mathbf{D y n}_{\mathbf{F}, X}$ we have

$$
a_{M^{Y}(v)}^{X}=\left.a_{M^{Y}(v)}^{Y}\right|_{X}=\left.a_{M^{Z}(v)}^{Z}\right|_{X}
$$

and as $\left(\mathcal{F}_{1}, \mathcal{T}_{1}^{X}\right) \in \mathbf{D y}_{\mathbf{F}, X}$ we have $a_{v}^{X}=\left.a_{v}^{Y}\right|_{X}=\left.a_{v}^{Z}\right|_{X}$. Thus we deduce that $m_{v}^{Y} \circ\left(m_{v}^{Z}\right)^{-1}$ fixes $\left.a_{M^{Z}(v)}^{Z}\right|_{X}$ which contains at least three elements so we have $m_{v}^{Y}=m_{v}^{Z}$.

According to this lemma we make an identification of $\mathbf{d y} \mathbf{n}_{\mathbf{F}, X}$ in $\widehat{\mathbf{r a t}}_{\mathbf{F}}$ and we define the topology of $\mathbf{d} \mathbf{y} \mathbf{n}_{\mathbf{F}, X}$ as the restriction of the one in $\widehat{\mathbf{r a t}}_{\mathbf{F}}$. With this topology we are going to prove the following.

Theorem 5.4. The space $\mathbf{d y n}_{\mathbf{F}, X}$ is compact.

5.3. Proof of compactness. We have $\operatorname{Quad}_{X} \subset \operatorname{Quad}_{Y}$. Denote by $\pi_{Y, X}$ the natural projection

$$
\pi_{Y, X}: \mathbb{S}^{\text {Quad }_{Y}} \rightarrow \mathbb{S}^{\text {QuadX }_{X}}
$$

Lemma 5.5. There exists a unique continuous map $\Pi_{Y, X}$ such that the following diagram commutes:

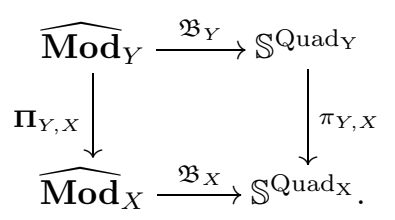

Proof. Let $\mathcal{T}^{Y}$ be a tree of spheres marked by $Y$. According to Lemma 3.12 if $\Pi_{Y, X}\left(\left[\mathcal{T}^{Y}\right]\right)$ exists, then it is unique.

Denote by $\mathcal{P}$ the collection of partitions of $X$ associated to the vertices of $T^{Y}$ separating three elements of $X$. This partition is independent of $\mathcal{T}^{Y} \in\left[\mathcal{T}^{Y}\right]$. Let us prove that the set $\mathcal{P}$ is an admissible collection of partitions (see Definition 3.3). 
1. By definition the vertices for which we are considering the partitions separate three elements of $X$.

2. Let $P$ be a partition corresponding to a vertex $v \in T^{Y}$ and $B \in P$. Either $B=\{x\}$, or $\operatorname{card} B>1$ and in this case, the branch on $V$ corresponding to $B$ contains at least an internal vertex separating two elements of $X$. Let $v^{\prime}$ be one of these vertices in this branch which are the closest to $v$ (for the length of $\left[v, v^{\prime}\right]$ ). Let $e^{\prime}$ be the edge on $v^{\prime}$ connecting $v$ to $v^{\prime}$. Then $B_{v^{\prime}}\left(e^{\prime}\right)=(X \backslash B)$. Indeed, suppose that this is not the case, we find an element $x \in B \cap B_{v^{\prime}}\left(e^{\prime}\right)$. Take $x_{1} \in B \backslash\{x\}$ and $x_{2} \in X \backslash B$. The vertex separating this triple $\left(x_{1}, x, x_{2}\right)$ is between $v$ and $v^{\prime}$ (because $x, x_{2} \in B_{v^{\prime}}\left(e^{\prime}\right)$ and $x, x_{1} \in B$ ) which contradicts the minimality of $v^{\prime}$.

3. Suppose by contradiction that we have $v_{1}$ and $v_{2}$ two vertices of $T^{Y}$ for which the associated partitions of $X$ are $P_{1}$ and $P_{2}$ and such that $P_{1} \cap P_{2} \ni B(\neq \emptyset)$. Let $B_{1}$ (resp. $B_{2}$ ) be the branch of $v_{1}$ (resp. $v_{2}$ ) corresponding to $B$. As $B \in B_{1} \cap B_{2}$ we have $v_{1} \in B_{2}$ (or $v_{1} \in B_{2}$ which is a symmetric case). Let $e_{1}$ be the edge on $v_{1}$ connecting it to $v_{2}$. Given that $v_{1}$ separate three elements of $X$, we find $x \in X \backslash\left(B \cup B_{v_{1}}\left(e_{1}\right)\right)$ which is absurd because $x \notin B_{v_{1}}\left(e_{1}\right)$ so $x \in B_{2} \in X=B$.

Hence $\mathcal{P}$ is an admissible collection of partitions and according to Corollary 3.6. the set $\mathcal{P}$ determines a unique isomorphism class of combinatorial trees $[T]$. For all $t \in$ Tripx, we denote by $v_{t}$ the vertex separating $t$ in $T^{Y}$. Denote by $\mathcal{T}$ the tree of spheres which combinatorial tree is the representative of $[T]$ for which each internal vertex associated to a triple $t$ is $v_{t}$ and for which the map associated to each internal vertex $v$ defined by a triple $t$ is $a_{v}:=\left.a_{v_{t}}\right|_{X}$. One can easily check that $\mathfrak{B}_{X}([\mathcal{T}])=\pi_{Y, X} \circ \mathfrak{B}_{Y}\left(\left[\mathcal{T}^{Y}\right]\right)$.

The continuity of the function $\boldsymbol{\Pi}_{Y, X}$ is clear from the definition of the topology on $\operatorname{Mod}_{Y}$ and $\operatorname{Mod}_{X}$.

Corollary 5.6. If $\mathcal{T}^{X}$ is compatible with $\mathcal{T}^{Y}$, then we have $\left[\mathcal{T}^{X}\right]=\Pi_{Y, X}\left(\left[\mathcal{T}^{Y}\right]\right)$. If $\Pi_{Y, X}\left(\left[\mathcal{T}^{Y}\right]\right)=[\mathcal{T}]$, then there exists a unique $\mathcal{T}^{X} \in[\mathcal{T}]$ compatible with $\mathcal{T}^{Y}$.

Proof. The first statement is clear. If $\Pi_{Y, X}\left(\left[\mathcal{T}^{Y}\right]\right)=[\mathcal{T}]$, we can choose a representative $\mathcal{T}^{X} \in[\mathcal{T}]$ whose set of vertices is included in the one of $T^{Y}$ and take the corresponding collection of spheres in $\mathcal{T}^{Y}$. We have to do this such that if a vertex $v \in T^{X} \cap T^{Y}$ separates three elements of $X$ in $T^{X}$, then it does the same in $T^{Y}$ and by definition of compatibility we also have to restrict the $a_{v}^{Y}$ to $X$. This determines a unique tree of spheres $\mathcal{T}^{X}$ in $[\mathcal{T}]$ compatible with $\mathcal{T}^{Y}$.

Remark 5.7. It follows that $\boldsymbol{\Pi}_{Y, X}$ is the natural quotient of a map $\tilde{\boldsymbol{\Pi}}_{Y, X}: \mathfrak{T}_{Y} \rightarrow \mathfrak{T}_{X}$. Heuristically, the map $\tilde{\boldsymbol{\Pi}}_{Y, X}$ consists in forgetting the vertices of the trees marked by $Y$ which are not separating three elements of $X$.

Proof. (Theorem 5.4) According to Proposition 4.2 and the definition of its topology, the set $\widehat{\mathbf{r a t}}_{\mathbf{F}}$ can be identified to a subspace of $\widehat{\operatorname{Mod}}_{Y} \times \widehat{\operatorname{Mod}}_{Z}$.

According to Corollary [5.6 and Lemma 5.5] we have

$$
\operatorname{dyn}_{\mathbf{F}, X}=\left\{\left(\left[\mathcal{T}^{Y}\right],\left[\mathcal{T}^{Z}\right]\right) \in \widehat{\operatorname{rat}}_{\mathbf{F}} \mid \boldsymbol{\Pi}_{Y, X}\left(\left[\mathcal{T}^{Y}\right]\right)=\boldsymbol{\Pi}_{Z, X}\left(\left[\mathcal{T}^{Z}\right]\right)\right\}
$$

So $\mathbf{d y n}_{\mathbf{F}, X}$ is a closed set in $\widehat{\mathbf{r a t}}_{\mathbf{F}}$ which is compact.

5.4. Convergence. In $\mathbf{D y n}_{\mathbf{F}, X}$ we define the natural convergence notion of a sequence in $\operatorname{Rat}_{\mathbf{F}, X}$ as follows. 
Definition 5.8 (Dynamical convergence). A sequence $\left(\mathcal{F}_{n}, a_{n}^{Y}, a_{n}^{Z}\right)_{n}$ in $\operatorname{Rat}_{\mathbf{F}, X}$ converges to $\left(\mathcal{F}, \mathcal{T}^{X}\right) \in \operatorname{Rat}_{\mathbf{F}, X}$ if

$$
\mathcal{F}_{n} \underset{\phi_{n}^{Y}, \phi_{n}^{Z}}{\longrightarrow} \mathcal{F} \quad \text { with } \quad \phi_{n, v}^{Y}=\phi_{n, v}^{Z}
$$

for all vertex $v$ internal vertex of $T^{X}$.

We prove that this dynamical convergence is compatible with the topology.

Lemma 5.9. A sequence of dynamical systems between marked spheres converges to a dynamical system between trees of spheres if and only if it dynamically converges to this limit.

Proof. Suppose that $\left(\mathcal{F}_{n}, \mathcal{T}_{n}^{X}\right)_{n}$ is a sequence of dynamical systems converging to a dynamical system $\left(\mathcal{F}, \mathcal{T}^{X}\right)$ :

$$
\mathcal{F}_{n} \underset{\left(\phi_{n}^{Y}, \phi_{n}^{Z}\right)}{\longrightarrow} \mathcal{F} \text {. }
$$

For all $t \in$ Tripx, we define $\tilde{\phi}_{n, t}^{Y}=\phi_{n, t}^{X}$ and $\tilde{\phi}_{n, t}^{Z}=\phi_{n, t}^{X}$ (see notation following Remark 3.17). Then, for all triple $t \in \operatorname{Trip}_{\mathrm{Y}}-\operatorname{Trip}_{\mathrm{X}}$, we define $\tilde{\phi}_{n, t}^{Y}=\phi_{n, t}^{Y}$ and for $t \in \operatorname{Tripz}_{\mathrm{Z}}-\operatorname{Trip}_{\mathrm{X}}, \tilde{\phi}_{n, t}^{Z}=\phi_{n, t}^{Z}$.

For all $t \in \operatorname{Trip}$ X,$\left(\tilde{\phi}_{n, t}^{Y}\right)^{-1} \circ \tilde{\phi}_{n, t}^{Y}$ tends to the identity of $\mathbb{S}_{t}$ because it converges to the identity on the three elements of $t$. Thus we have $\left(\mathcal{F}_{n}, \mathcal{T}_{n}^{X}\right)$ converges dynamically to $\left(\mathcal{F}, \mathcal{T}^{X}\right)$ with respect to the families of sequences $\left(\tilde{\phi}_{n}^{Y}\right)_{n}$ and $\left(\tilde{\phi}_{n}^{Z}\right)_{n}$.

We can now deduce the statement below that was assumed in A1.

Corollary 5.10. If $\left(\mathcal{F}_{n}\right)_{n}$ is a sequence in $\operatorname{Rat}_{\mathbf{F}, X}$, then after passing to a subsequence, there exists $\left(\mathcal{F}, \mathcal{T}^{X}\right) \in \mathbf{D y}_{\mathbf{F}, X}$ such that $\left(\mathcal{F}_{n}, \mathcal{T}_{n}^{X}\right)_{n}$ converges dynamically to $\left(\mathcal{F}, \mathcal{T}^{X}\right)$.

Proof. This corollary follows directly from Theorem 5.4 and Lemma 5.9.

Proposition 5.11. We have the following inclusions:

$$
\overline{\operatorname{rat}}_{\mathbf{F}, X} \subsetneq \mathbf{d y n} \mathbf{F , X} \subsetneq \widehat{\operatorname{rat}}_{\mathbf{F}} .
$$

Proof. In A2 we give an example of element in $\mathbf{D} \mathbf{y} \mathbf{n}_{\mathbf{F}, X}$ that is not a dynamical limit of dynamical covers between marked spheres so we have $\overline{\operatorname{rat}_{\mathbf{F}, X}} \subsetneq \mathbf{d} \mathbf{y} \mathbf{n}_{\mathbf{F}, X}$.

Remark 5.12. In A2 are proven several general properties about dynamical systems between trees of spheres which are limits of dynamical systems between marked spheres called the annuli lemmas (Lemma 3.3) and branch lemma (Lemma 3.1).

\section{ACKNOWLEDGMENTS}

I would like to thank my advisor, Xavier Buff, for the time he spent teaching me how to write and make clear my ideas. Thanks go to Jan Kiwi and Laura De Marco for helpful comments. Thanks go also to the anonymous referee, especially for introducing me to the [FT]. 


\section{REFERENCES}

[A] M. Arfeux, Dynamique holomorphe et arbres de sphères, Thèse de l'université Toulouse.

[A1] M. Arfeux, Dynamics on Trees of Spheres, Journal of the London Math. Soc. 95 (2017), no. 1, 177-202, DOI 10.1112/jlms.12016.

[A2] M. Arfeux, Approximability of dynamical systems between trees of spheres, Indiana Univ. Math. J. 65 (2016), no. 6, 1945-1977, DOI 10.1512/iumj.2016.65.5823. MR.3595486

[B] L. Bers, On spaces of Riemann surfaces with nodes, Bull. Amer. Math. Soc. 80 (1974), 1219-1222, DOI 10.1090/S0002-9904-1974-13686-4. MR0361165

[DeM] P. Deligne and D. Mumford, The irreducibility of the space of curves of given genus, Inst. Hautes Études Sci. Publ. Math. 36 (1969), 75-109. MR0262240

[Di] R. Diestel, Graph theory, 3rd ed., Graduate Texts in Mathematics, vol. 173, SpringerVerlag, Berlin, 2005. MR2159259

[FT] R. Funahashi and M. Taniguchi, The cross-ratio compactification of the configuration space of ordered points on $\widehat{\mathbb{C}}$, Acta Math. Sin. (Engl. Ser.) 28 (2012), no. 10, 2129-2138, DOI 10.1007/s10114-012-1185-x. MR2966959

Pontificia Universidad Católica de Valparaíso, Blanco Viel 596, Cerro Barón, ValPARAíso, Chile

E-mail address: matthieu.arfeux@pucv.cl 